\title{
Escape rates for Gibbs measures
}

\author{
ANDREW FERGUSON and MARK POLLICOTT \\ Mathematics Institute, Zeeman Building, University of Warwick, Coventry CV4 7AL, UK \\ (e-mail:a.j.ferguson@warwick.ac.uk,m.pollicott@warwick.ac.uk)
}

(Received 30 August 2010 and accepted in revised form 4 January 2011)

Abstract. In this paper we study the asymptotic behaviour of the escape rate of a Gibbs measure supported on a conformal repeller through a small hole. There are additional applications to the convergence of the Hausdorff dimension of the survivor set.

\section{Introduction}

Given any transformation $T: X \rightarrow X$ preserving an ergodic probability measure $\mu$ and any Borel set $A \subset X$, the escape rate quantifies the asymptotic behaviour of the measure of the set of points $x \in X$ for which none of the first $n$ terms in the orbit intersects $U$. Bunimovich and Yurchenko [7] considered the fundamental case of the doubling map and Haar measure, and where $U$ is a dyadic interval. Subsequently, Keller and Liverani [16] proved a more general perturbation result which was then used to show, amongst other things, that a similar formula holds in the case that $T$ is an expanding interval map and $\mu$ the absolutely continuous invariant probability measure. Other papers regarding this topic include $[1,6,9,19]$ and references therein.

In this paper, we prove analogous results in the more general setting of Gibbs measures supported on conformal repellers. Much of the analysis is undertaken in the setting of subshifts of finite type; this not only allows us to prove similar results for a broad class of maps which can be modelled symbolically but also improve on the work of Lind [18] who considered the convergence of topological entropy for a topologically mixing subshift.

Another interesting aspect of our analysis is the connection with the work of Hirata [12] on the exponential law for first return times for Axiom A diffeomorphisms. Some of the ingredients in our approach were suggested by Hirata's paper, although we had to significantly modify the actual details.

Let $\mathcal{M}$ be a Riemannian manifold and $f: \mathcal{M} \rightarrow \mathcal{M}$ a $C^{1}$-map. Let $J$ be a compact subset of $\mathcal{M}$ such that $f(J)=J$. We say that the pair $(J, f)$ is a conformal repeller if:

(1) $\left.f\right|_{J}$ is a conformal map;

(2) there exist $c>0$ and $\lambda>1$ such that $\left\|d f_{x}^{n} v\right\| \geq c \lambda^{n}\|v\|$ for all $x \in J, v \in T_{x} \mathcal{M}$, and $n \geq 1$

(3) $\quad f$ is topologically mixing on $J$; 
(4) $J$ is maximal, i.e. there exists an open neighbourhood $V \supset J$ such that

$$
J=\left\{x \in V: f^{n}(x) \in V \text { for all } n \geq 0\right\} .
$$

Let $\phi: J \rightarrow \mathbb{R}$ be $\alpha$-Hölder and let $\mu$ denote the associated equilibrium state, i.e.

$$
P(\phi)=\sup \left\{h_{v}(f)+\int \phi d v: f_{*}(v)=v, v(J)=1\right\}=h_{\mu}(f)+\int \phi d \mu,
$$

where $h_{v}(f)$ denotes the Kolmogorov-Sinai entropy of the measure $v$ (see [26] for further details).

Fix $z \in J$; for $\epsilon>0$, we define the escape rate of $\mu$ through $B(z, \epsilon)$ (i.e. the rate at which mass 'escapes' or 'leaks' through the hole $B(z, \epsilon))$ by

$$
r_{\mu}(B(z, \epsilon))=-\lim \sup _{k \rightarrow \infty} \frac{1}{k} \log \mu\left\{x \in J: f^{i}(x) \notin B(z, \epsilon), 0 \leq i \leq k-1\right\} .
$$

Our first result concerns the asymptotic behaviour of $r_{\mu}(B(z, \epsilon))$ for small $\epsilon$.

THEOREM 1.1. Let $(J, f)$ be a conformal repeller, $\phi: J \rightarrow \mathbb{R}$ Hölder continuous, and $\mu$ the associated equilibrium state. Fix $z \in J$; then

$$
\lim _{\epsilon \rightarrow 0} \frac{r_{\mu}(B(z, \epsilon))}{\mu(B(z, \epsilon))}=d_{\phi}(z)= \begin{cases}1 & \text { if } z \text { is not periodic }, \\ 1-e^{\phi^{p}(z)-p P(\phi)} & \text { if } z \text { has prime period } p,\end{cases}
$$

where $\phi^{p}(z)=\phi(z)+\phi(f(z))+\cdots+\phi\left(f^{p-1}(z)\right)$.

We also obtain an asymptotic formula for the Hausdorff dimension of the survivor set:

$$
J_{\epsilon}=\left\{x \in J: f^{k}(x) \notin B(z, \epsilon) \text { for all } k \geq 0\right\},
$$

i.e. all points whose orbits are $\epsilon$-bounded away from $z$.

Suppose now that $f \in C^{1+\alpha}(J)$ for some $\alpha>0$. Let $\mu$ denote the equilibrium state related to the potential $\phi=-s \log \left|f^{\prime}\right|$, where $s=\operatorname{dim}_{H}(J)$. For $\epsilon>0$, we let $s_{\epsilon}$ denote the Hausdorff dimension of the set $J_{\epsilon}$.

THEOREM 1.2. Let $(J, f)$ be a conformal repeller with $f \in C^{1+\alpha}(J)$. Let $\phi=-s \log \left|f^{\prime}\right|$ and let $\mu$ denote the associated equilibrium state. Fix $z \in J$; then

$$
\lim _{\epsilon \rightarrow 0} \frac{s-s_{\epsilon}}{\mu(B(z, \epsilon))}=\frac{d_{\phi}(z)}{\int \log \left|f^{\prime}\right| d \mu} .
$$

Remark 1.3. A similar formula was obtained by Hensley [11] in the setting of continued fractions.

The paper is structured as follows: in $\S 2$ we apply Theorems 1.1 and 1.2 to concrete examples. In $\S 3$ we study the spectral properties of transfer operators acting on a certain class of Banach spaces. Section 4 contains a perturbation result, while in $\S 5$ we prove the result in the analogue of Theorem 1.1 in the setting of subshifts of finite type. Finally, $\S \S 6$ and 7 contain the proofs of Theorems 1.1 and 1.2, respectively.

\section{Examples}

To illustrate the main results, we briefly consider two simple examples.

2.1. Hyperbolic Julia sets. Let $f: \widehat{\mathbb{C}} \rightarrow \widehat{\mathbb{C}}$ be a rational map of degree $d \geq 2$, where $\widehat{\mathbb{C}}$ denotes the Riemann sphere. The Julia set of $R$ is the closure of the repelling periodic 
points of $f$, i.e.

$$
J=\operatorname{cl}\left(\left\{z \in \widehat{\mathbb{C}}: f^{p}(z)=z \text {, for some } p \geq 1 \text { and }\left|\left(f^{p}\right)^{\prime}(z)\right|>1\right\}\right) .
$$

The map $f: J \rightarrow J$ is a conformal expanding map and the results of the previous section apply. As an example, the map $f(z)=z^{2}+c$ for $|c|<1 / 4$ is hyperbolic. Define $\phi: J \rightarrow \mathbb{R}$ by $\phi(z)=-s \log |2 z|$, where $s$ denotes the Hausdorff dimension of $J$. Let $\mu$ denote the associated equilibrium state. Setting $z=(1+\sqrt{1-4 c}) / 2$, we see that $f(z)=z$ and $\left|f^{\prime}(z)\right|>1$ and accordingly Theorem 1.1 implies that

$$
\lim _{\epsilon \rightarrow 0} \frac{r_{\mu}(B(z, \epsilon))}{\mu(B(z, \epsilon))}=1-\frac{1}{|2 z|^{s}} .
$$

2.2. One-dimensional Markov maps. Assume that there exist a finite family of disjoint closed intervals $I_{1}, I_{2}, \ldots, I_{m} \subset[0,1]$ and a $C^{1+\alpha}$-map $f: \bigcup_{i} I_{i} \rightarrow[0,1]$ such that:

(1) for every $i$, there is a subset $P=P(i)$ of indices with $f\left(I_{i}\right) \cap \bigcup_{i} I_{i}=\bigcup_{k \in P} I_{k}$;

(2) for every $x \in \bigcup_{i} \operatorname{int}\left(I_{i}\right)$, the derivative of $f$ satisfies $\left|f^{\prime}(x)\right| \geq \rho$ for some fixed $\rho>0$;

(3) there exist $\lambda>1$ and $n_{0}>0$ such that if $f^{m}(x) \in \bigcup_{i} I_{i}$ for all $0 \leq m \leq n_{0}-1$, then $\left|\left(f^{n_{0}}\right)^{\prime}(x)\right| \geq \lambda$.

Let $J=\left\{x \in[0,1]: f^{n}(x) \in \bigcup_{i} I_{i}\right.$ for all $\left.n \in \mathbb{N}\right\}$. The set $J$ is a repeller for the map $f$ and conformality follows from the domain being one dimensional.

If we take $I_{1}=[0,1 / 3], I_{2}=[2 / 3,1]$, and let $f(x)=3 x(\bmod 1)$, the associated repeller $J$ is the middle-third Cantor set. Let $z=1 / 4$; then $z \in J$ and has prime period 2 . Set $\phi(x)=-\log (2)$ and let $\mu$ denote the associated equilibrium state. Then Theorem 1.1 implies that

$$
\lim _{\epsilon \rightarrow 0} \frac{r_{\mu}(B(1 / 4, \epsilon))}{\mu(B(1 / 4, \epsilon))}=1-\frac{1}{2^{2}}=\frac{3}{4} .
$$

For $\epsilon>0$, we set

$$
J_{\epsilon}=\left\{x \in J: f^{k}(x) \notin B(1 / 4, \epsilon) \text { for } k=0,1,2, \ldots\right\} .
$$

Let $s_{\epsilon}=\operatorname{dim}_{H}\left(J_{\epsilon}\right)$ and $s=\log (2) / \log (3)$; then Theorem 1.2 implies that

$$
\lim _{\epsilon \rightarrow 0} \frac{s-s_{\epsilon}}{\mu(B(1 / 4, \epsilon))}=\frac{3}{4 \log (3)} .
$$

\section{Spectral properties of the transfer operator}

In this section, we study the spectral properties of the transfer operator. We first fix notation which will be used for the rest of the paper. Throughout the rest of the paper, $c$ will denote a positive and finite constant which may change in value with successive uses. Let $A$ denote an irreducible and aperiodic $l \times l$ matrix of zeroes and ones, i.e. there exists a positive integer $d$ such that $A^{d}>0$. We define the subshift of finite type (associated with matrix $A$ ) to be

$$
\Sigma=\left\{\left(x_{n}\right)_{n=0}^{\infty}: A\left(x_{n}, x_{n+1}\right)=1 \text { for all } n\right\} .
$$

If we equip the set $\{0,1, \ldots, l-1\}$ with the discrete topology, then $\Sigma$ is compact in the corresponding Tychonov product topology. The shift $\sigma: \Sigma \rightarrow \Sigma$ is defined by $\sigma(x)=y$, where $y_{n}=x_{n+1}$ for all $n$, i.e. the sequence is shifted one place to the left and the first entry deleted. 
For $\theta \in(0,1)$, we define a metric on $\Sigma$ by $d_{\theta}(x, y)=\theta^{m}$, where $m$ is the least positive integer (assuming that such an $m$ exists) with $x_{m} \neq y_{m}$; otherwise, we set $d_{\theta}(x, x)=0$. Equipped with the metric $d_{\theta}$, the space $\left(\Sigma, d_{\theta}\right)$ is complete, and moreover the topology induced by $d_{\theta}$ agrees with the previously mentioned Tychonov product topology. Finally, for $x \in \Sigma$ and a positive integer $n \geq 1$, we define the cylinder of length $n$ centred on $x$ to be the set

$$
[x]_{n}=\left[x_{0}, x_{1}, \ldots, x_{n-1}\right]=\left\{y \in \Sigma: y_{i}=x_{i} \text { for } i=0,1, \ldots, n-1\right\} .
$$

Fix a $d_{\theta}$-Lipschitz continuous function $\phi: \Sigma \rightarrow \mathbb{R}$ and recall that we let $\mu$ denote its equilibrium state defined in the introduction, i.e.

$$
P(\phi):=\sup \left\{h_{v}+\int \phi d \nu: \sigma_{*}(\nu)=v, v(\Sigma)=1\right\}=h_{\mu}+\int \phi d \mu .
$$

We let

$$
L^{1}(\mu):=\left\{w: \Sigma \rightarrow \mathbb{C}: w \text { is measurable and } \int|w| d \mu<\infty\right\},
$$

which, equipped with the norm $\|w\|_{1}=\int|w| d \mu$, is a Banach space. We now describe a particular subspace of $L^{1}(\mu)$ on which the transfer operator will act: for $w \in L^{1}(\mu)$, $x \in \Sigma$, and a positive integer $m$, we set

$$
\operatorname{osc}(w, m, x)=\operatorname{esssup}\left\{|w(y)-w(z)|: y, z \in[x]_{m}\right\} .
$$

We introduce the semi-norm

$$
|w|_{\theta}=\sup _{m \geq 1} \theta^{-m}\|\operatorname{osc}(w, m, \cdot)\|_{1} .
$$

We let

$$
\mathcal{B}_{\theta}=\left\{w \in L^{1}(\mu):|w|_{\theta}<\infty\right\}
$$

It is worth noting that if we were to take the supremum norm $\|\cdot\|_{\infty}$ in place of the $L^{1}$ norm, then the space coincides with Lipschitz continuous functions (with respect to the metric $d_{\theta}$ ).

We equip $B_{\theta}$ with the norm

$$
\|w\|_{\theta}=|w|_{\theta}+\|w\|_{1} .
$$

This space was first introduced by Keller [14], in a more general framework, where the following result was also proved.

Proposition 3.1. (Keller) The space $\left(\mathcal{B}_{\theta},\|\cdot\|_{\theta}\right)$ is complete. Furthermore, the set $\left\{w \in \mathcal{B}_{\theta}:\|w\|_{\theta} \leq c\right\}$ is $L^{1}$-compact for any $c>0$.

We introduce the transfer operator $\mathcal{L}=\mathcal{L}_{\phi}: \mathcal{B}_{\theta} \rightarrow \mathcal{B}_{\theta}$

$$
(\mathcal{L} w)(x)=\sum_{\sigma(y)=x} e^{\phi(y)} w(y) .
$$

We let $i=\left(i_{0}, i_{1}, \ldots, i_{k-1}\right)$ denote an allowed string of length $k$; then we can write $\left(\mathcal{L}^{k} w\right)(x)=\sum_{|i|=k} e^{\phi^{k}(i x)} w(i x)$, where the sum is over those strings for which the concatenation $i x$ is allowed, i.e. we require that $i x \in \Sigma$. 
Another Banach space that we require is that of Lipschitz functions

$$
\mathcal{F}_{\theta}=\left\{w: \Sigma \rightarrow \mathbb{C}: \sup _{m \geq 1} \theta^{-m}\|\operatorname{osc}(w, m, \cdot)\|_{\infty}<\infty\right\} .
$$

The following theorem describes the spectral properties of $\mathcal{L}$ acting on the space $\mathcal{F}_{\theta}$ of $d_{\theta}$-Lipschitz continuous functions; for a proof, see [21, Theorem 2.2].

Proposition 3.2. (Ruelle) Let $\phi \in \mathcal{F}_{\theta}$ be real valued and suppose that $A$ is irreducible and aperiodic.

(1) There is a simple maximal positive eigenvalue $\lambda=\lambda_{\phi}$ of $\mathcal{L}$ with corresponding strictly positive eigenfunction $g=g_{\phi} \in \mathcal{F}_{\theta}$.

(2) The remainder of the spectrum of $\mathcal{L}: \mathcal{F}_{\theta} \rightarrow \mathcal{F}_{\theta}$ (excluding $\lambda>0$ ) is contained in a disk of radius strictly smaller than $\lambda$.

(3) There is a unique probability measure $v$ such that $\mathcal{L}^{*} v=\lambda v$.

(4) $\lambda^{-k} \mathcal{L}^{k} w \rightarrow g \int w d v$ uniformly for all $w \in \mathcal{F}_{\theta}$, where $g$ is as above and $\int g d v=1$.

We remark that the equilibrium state $\mu$ is absolutely continuous with respect to the eigenmeasure $v$, with the Radon-Nikodym derivative being given by the eigenfunction $g$. By scaling the operator $\mathcal{L}$, if necessary, we may assume without loss of generality that $\lambda=1$; further, as $g>0$, we may assume that $\mathcal{L} 1=1$.

Another useful property of $\mu$ is the Gibbs property (see [3] for further details). Namely, there exists a constant $c>1$ such that for any $x \in \Sigma$ and positive integer $n$ we have that

$$
c^{-1} \leq \frac{\mu[x]_{n}}{e^{\phi^{n}(x)}} \leq c .
$$

We now prove a result relating to the spectrum of $\mathcal{L}$ acting on $\mathcal{B}_{\theta}$, namely that it has a spectral gap. A crucial part in this process is proving a Lasota-Yorke inequality $\dagger$.

LEMMA 3.3. There exists $c>0$ such that for any $w \in \mathcal{B}_{\theta}$ we have

$$
\left|\mathcal{L}^{k} w\right|_{\theta} \leq c\left(\theta^{k}|w|_{\theta}+\|w\|_{1}\right) \text {. }
$$

Proof. Let $x, y \in \Sigma$ be such that $d_{\theta}(x, y) \leq \theta^{m}$; then

$$
\begin{aligned}
\left|\mathcal{L}^{k} w(x)-\mathcal{L}^{k} w(y)\right| & \leq \sum_{|i|=k}\left|e^{\phi^{k}(i x)} w(i x)-e^{\phi^{k}(i y)} w(i y)\right| \\
& \leq \sum_{|i|=k} e^{\phi^{k}(i x)} \operatorname{osc}(w, k+m, i x)+e^{\phi^{k}(i x)}\left|1-e^{\phi^{k}(i y)-\phi^{k}(i x)}\right||w(i y)| \\
& \leq c \sum_{|i|=k}\left(e^{\phi^{k}(i x)} \operatorname{osc}(w, k+m, i x)+\theta^{m} \frac{e^{\phi^{k}(i x)}}{\mu[i x]_{k+m}} \int_{[i x]_{k+m}}|w| d \mu\right) \\
& \leq c \sum_{|i|=k}\left(e^{\phi^{k}(i x)} \operatorname{osc}(w, k+m, i x)+\theta^{m} \frac{c}{\mu[x]_{m}} \int_{[i x]_{k+m}}|w| d \mu\right),
\end{aligned}
$$

where we used the Gibbs property (1) in the final line, i.e.

$$
\frac{e^{\phi^{k}(i x)}}{\mu[i x]_{k+m}} \leq \frac{c}{e^{\phi^{m}(x)}} \leq \frac{c^{2}}{\mu[x]_{m}} .
$$

$\dagger$ The term 'Lasota-Yorke' refers to the modern usage dating back to their paper [17]. Similar inequalities date back to Ionescu Tulcea-Marinescu [13] and perhaps earlier. 
Thus,

$$
\operatorname{osc}\left(\mathcal{L}^{k} w, m, x\right) \leq c \sum_{|i|=k}\left(e^{\phi^{k}(i x)} \operatorname{osc}(w, k+m, i x)+\theta^{m} \frac{c}{\mu[x]_{m}} \int_{[i x]_{k+m}}|w| d \mu\right) .
$$

Integrating with respect to $\mu$, and again invoking (1), we see that

$$
\int \operatorname{osc}\left(\mathcal{L}^{k} w, m, x\right) d \mu(x) \leq c\left(\int \operatorname{osc}(w, k+m, x) d \mu(x)+\theta^{m}\|w\|_{1}\right) ;
$$

dividing by $\theta^{m}$ and taking suprema yields

$$
|\mathcal{L} w|_{\theta} \leq c\left(\theta^{k}|w|_{\theta}+\|w\|_{1}\right)
$$

Finally, we see that

$$
\begin{aligned}
\left\|\mathcal{L}^{k} w\right\|_{\theta} & =\left|\mathcal{L}^{k} w\right|_{\theta}+\left\|\mathcal{L}^{k} w\right\|_{1} \\
& \leq c \theta^{k}\left(|w|_{\theta}+\|w\|_{1}\right)+\|w\|_{1} \\
& \leq c\left(\theta^{k}|w|_{\theta}+\|w\|_{1}\right) .
\end{aligned}
$$

Lemma 3.4. The operator $\mathcal{L}: \mathcal{B}_{\theta} \rightarrow \mathcal{B}_{\theta}$ has a simple maximal eigenvalue $\lambda=1$, while the rest of the spectrum is contained in a ball of radius strictly less than 1.

Proof. We begin by proving that for any $w \in \mathcal{B}_{\theta}, \mathcal{L}^{k} w$ converges to $\int w d \mu$ in $L^{1}(\mu)$. Fix $\epsilon>0$ and choose $v \in \mathcal{F}_{\theta}$ such that $\|v-w\|_{1}<\epsilon / 3$; by Proposition 3.2, there exists a positive integer $N$ such that $\left\|\mathcal{L}^{n}(v)-\int v d \mu\right\|_{1}<\epsilon / 3$ for all $n \geq N$, in which case we see that

$$
\begin{aligned}
\left\|\mathcal{L}^{n}(w)-\int w d \mu\right\|_{1} & \leq\left\|\mathcal{L}^{n}(w-v)\right\|_{1}+\left\|\mathcal{L}^{n}(v)-\int v d \mu\right\|_{1}+\left\|\int v d \mu-\int w d \mu\right\|_{1} \\
& \leq 2\|v-w\|_{1}+\left\|\mathcal{L}^{n}(v)-\int v d \mu\right\|_{1}<\epsilon .
\end{aligned}
$$

This in turn implies that for each $w \in B=\left\{v \in \mathcal{B}_{\theta}:\|v\|_{\theta} \leq 1\right\}$,

$$
\left\|\left.\mathcal{L}^{n}(w)\right|_{\mathbb{C}^{\perp}}\right\|_{1}=\inf _{c \in \mathbb{C}}\left\|\mathcal{L}^{n}(w)-c\right\|_{1} \rightarrow 0 \quad \text { as } n \rightarrow \infty,
$$

where $\mathbb{C}^{\perp}=\left\{w \in \mathcal{B}_{\theta}: \int w d \mu=0\right\}$. We claim that this convergence is uniform over $B$. To see this, fix $\delta>0$ and $w \in B$; then there exists a positive integer $N=N(w)$ such that $\left\|\left.\mathcal{L}^{n}(w)\right|_{\mathbb{C}^{\perp}}\right\|_{1} \leq \delta / 2$ for all $n \geq N$. By Proposition 3.1, $B$ is compact and so the cover $\left\{B_{1}(w, \delta / 2)\right\}_{w \in B}$ has a finite subcover, say $B_{1}\left(w_{1}, \delta / 2\right), B_{1}\left(w_{2}, \delta / 2\right), \ldots, B_{1}\left(w_{m}, \delta / 2\right)$. In which case, if $n \geq N:=\max _{i=1,2, \ldots, m} N\left(w_{i}\right)$, we have $\left\|\left.\mathcal{L}_{\phi}^{n}(w)\right|_{\mathbb{C}^{\perp}}\right\|_{1} \leq \delta$ for any $w \in B$.

Finally, to show the existence of a spectral gap, from Proposition 3.3, we observe for $w \in B$ and $n \geq N$ that

$$
\begin{aligned}
& \left\|\left.\mathcal{L}^{2 n}(w)\right|_{\mathbb{C}^{\perp}}\right\|_{\theta} \leq c\left(\left.\theta^{n}\left|\mathcal{L}^{n}(w)\right|_{\mathbb{C}^{\perp}}\right|_{\theta}+\left\|\left.\mathcal{L}^{n}(w)\right|_{\mathbb{C}^{\perp}}\right\|_{1}\right) \\
& \leq c\left(\left.\theta^{2 n}|w|_{\mathbb{C}^{\perp}}\right|_{\theta}+\theta^{n}\left\|\left.w\right|_{\mathbb{C}^{\perp}}\right\|_{1}+\left\|\left.\mathcal{L}^{n}(w)\right|_{\mathbb{C}^{\perp}}\right\|_{1}\right) \\
& \leq c\left(\theta^{2 n}+\theta^{n}+\delta\right) \text {. }
\end{aligned}
$$

We may choose $n$ and $\delta$ so that $\left\|\left.\mathcal{L}^{2 n}(w)\right|_{\mathbb{C}^{\perp}}\right\|_{\theta}<1$, which proves that $\mathcal{L}$ has a spectral gap. 
3.1. Singular perturbations of the transfer operator. We introduce a perturbation of the transfer operator $\mathcal{L}$ : let $\left\{U_{n}\right\}_{n}$ be a family of open sets; further, we require that they satisfy the following technical conditions:

(1) $\left\{U_{n}\right\}_{n}$ are nested with $\bigcap_{n \geq 1} U_{n}=\{z\}$;

(2) each $U_{n}$ consists of a finite union of cylinder sets, with each cylinder having length $n$;

(3) there exist constants $c>0,0<\rho<1$ such that $\mu\left(U_{n}\right) \leq c \rho^{n}$ for $n \geq 1$;

(4) there are a sequence $\left\{l_{n}\right\}_{n} \subset \mathbb{N}$ and a constant $\kappa>0$ such that $\kappa<l_{n} / n \leq 1$ and $U_{n} \subset[z]_{l_{n}}$ for all $n \geq 1$;

(5) if $\sigma^{p}(z)=z$ has prime period $p$, then $\sigma^{-p}\left(U_{n}\right) \cap\left[z_{0} z_{1} \cdots z_{p-1}\right] \subseteq U_{n}$ for large enough $n$.

Remark 3.5. We observe that (5) above is not absolutely essential for the application to conformal repellers and serves only to greatly simplify the analysis.

For $n \geq 1$, we define the perturbed operator $\mathcal{L}_{n}: \mathcal{B}_{\theta} \rightarrow \mathcal{B}_{\theta}$ by

$$
\mathcal{L}_{n}(w)(x)=\mathcal{L}\left(\chi_{U_{n}^{c}} w\right)(x) .
$$

For a positive integer $n$, we let $\Sigma_{n}=\bigcap_{k \geq 0} \Sigma \backslash \sigma^{-j}\left(U_{n}\right)$. By choosing $n$ large enough, we can ensure that the system $\left(\Sigma_{n},\left.\sigma\right|_{\Sigma_{n}}\right)$ is topologically mixing, and so the results of [8] apply, namely we have the following proposition.

Proposition 3.6. (Collet, Martínez, Schmitt) For each $n$, there exist a continuous $g_{n}$ : $\Sigma \rightarrow \mathbb{R}$ with $g_{n}>0$ and a $\lambda_{n}>0$ such that $\mathcal{L}_{n} g_{n}=\lambda_{n} g_{n}$; moreover, for any continuous $w: \Sigma \rightarrow \mathbb{C}$, we have

$$
\left\|\lambda_{n}^{-k} \mathcal{L}_{n}^{k} w-v_{n}\left(\left.w\right|_{\Sigma_{n}}\right) g_{n}\right\|_{\infty} \rightarrow 0
$$

where $v_{n}$ denotes the unique probability measure guaranteed by Proposition 3.2, i.e. $v_{n}$ satisfies $\operatorname{supp}\left(v_{n}\right)=\Sigma_{n}$ and $\left(\mathcal{L}_{n}^{*} v_{n}\right)(w)=\lambda_{n} v_{n}(w)$ for $w \in \mathcal{F}_{\theta}\left(\Sigma_{n}\right)$.

Moreover, we may prove a Lasota-Yorke style inequality for $\mathcal{L}_{n}: \mathcal{B}_{\theta} \rightarrow \mathcal{B}_{\theta}$, which, in conjunction with Proposition 3.6 and the methods of Lemma 3.4, we can use to show that $g_{n} \in \mathcal{B}_{\theta}$ and that $\lambda_{n}$ is a simple maximal eigenvalue for $\mathcal{L}_{n}: \mathcal{B}_{\theta} \rightarrow \mathcal{B}_{\theta}$.

The perturbation $\mathcal{L}_{n}$ is singular with respect to the $\|\cdot\|_{\theta}$ norm. We adopt the approach of [15] and introduce a weak norm

$$
\|w\|_{h}:=|w|_{h}+\|w\|_{1}=\sup _{j \geq 0} \sup _{m \geq 1} \theta^{-m} \int_{\sigma^{-j}\left(U_{m}\right)}|w| d \mu+\|w\|_{1} .
$$

Throughout this section, we assume that $\theta \in(\rho, 1)$. Our first result states that the weak norm is dominated by the strong norm.

LEMMA 3.7. Under the assumptions above, we have

$$
\|w\|_{h} \leq c\|w\|_{\theta}
$$

for all $w \in \mathcal{B}_{\theta}$. 
Proof. We first relate the strong norm with the $L^{\infty}$ norm. Let $c=\max _{i=0,1, \ldots, l-1} \mu[i]_{1}^{-1}$; then, for $\mu$ almost all $x \in \Sigma$,

$$
\begin{aligned}
|w(x)| & \leq \operatorname{osc}(w, 1, x)+c \int_{\left[x_{0}\right]_{1}}|w| d \mu \\
& \leq c\left(\int_{\left[x_{0}\right]_{1}} \operatorname{osc}(w, 1, y) d \mu(y)+\int_{\left[x_{0}\right]_{1}}|w| d \mu\right) \\
& \leq c\|w\|_{\theta} .
\end{aligned}
$$

If $\theta \in(\rho, 1)$, then

$$
|w|_{h} \leq \sup _{m \geq 1} \theta^{-m} \mu\left(U_{m}\right)\|w\|_{\infty} \leq c\|w\|_{\theta}
$$

3.2. Convergence of the spectral radii. In this section, we prove a preliminary result relating to the behaviour of the spectra of the operators $\mathcal{L}_{n}$ acting on $\mathcal{B}_{\theta}$. From Proposition 3.6, it is easy to see that for any $u \in \Sigma$ we have

$$
P_{\Sigma_{n}}(\phi):=\log \lambda_{n}=\lim _{k \rightarrow \infty} \frac{1}{k} \log \left(\mathcal{L}_{n}^{k} 1(u)\right) .
$$

Proposition 3.8. Under assumptions (1)-(5), we have $\lim _{n \rightarrow \infty} \lambda_{n}=\lambda$.

Proof. As $U_{n} \subset[z]_{l_{n}}$, setting $\tilde{\Sigma}_{n}=\Sigma \backslash \bigcap_{k \geq 0} \sigma^{-k}[z]_{l}$, it is easy to see that $\tilde{\Sigma}_{n} \subset \Sigma_{n}$. Accordingly, it suffices to show that $P_{\tilde{\Sigma}_{n}}(\phi) \rightarrow P(\phi)$.

As $(\Sigma, \sigma)$ is topologically mixing, we may find a positive integer $d$ such that $A^{d}>0$. Fix $u \in \Sigma$; for integers $k$ and $n$, we set

$$
\begin{gathered}
B_{k}=\left\{x_{0} x_{1} \cdots x_{k-1}: x_{0} x_{1} \cdots x_{k-1} u \in \Sigma\right\}, \\
Z_{k}(\phi)=\sum_{x_{0} x_{1} \cdots x_{k-1} \in B_{k}} e^{\phi^{k}\left(x_{0} x_{1} \cdots x_{k-1} u\right)}, \quad Z_{k, n}(\phi)=\sum_{x_{0} x_{1} \cdots x_{k-1} \in B_{k, n}} e^{\phi^{k}\left(x_{0} x_{1} \cdots x_{k-1} u\right)} .
\end{gathered}
$$

It is easy to see that $\mathcal{L}^{k} 1(u)=Z_{k}(\phi)$ (respectively $\left.\mathcal{L}_{n}^{k} 1(u)=Z_{k, n}(\phi)\right)$ and so by (2) we have that $P(\phi)=\lim _{k \rightarrow \infty}(1 / k) \log Z_{k}(\phi)$ (respectively $P_{\Sigma_{n}}(\phi)=\lim _{k \rightarrow \infty}(1 / k)$ $\left.\log Z_{k, n}(\phi)\right)$.

Fix $\epsilon>0$. By (3), there exists $a>0$ such that $Z_{k}(\phi) \geq a e^{k(P(\phi)-\epsilon)}$ for all $k \geq 1$. In addition, as $h_{\text {top }}(\sigma)>0$, there exists $b>0$ such that $\left|B_{k}\right| \geq b e^{k\left(h_{\text {top }}(\sigma)-\epsilon\right)}$ for all $k \geq 1$.

Fix large integers $k$ and $n$ such that both $b e^{k\left(h_{\mathrm{top}}(\sigma)-\epsilon\right)}>l_{n}-k+1$ and $2(k+d)<$ $l_{n} \epsilon$. Observe that the string $z_{0} z_{1} \cdots z_{l_{n-1}}$ has precisely $l_{n}-k+1$ subwords of length $k$; accordingly, the first condition on $k$ and $n$ guarantees the existence of a finite word $x \in B_{k}$ such that $x$ does not appear as a subword of $z_{0} z_{1} \cdots z_{l_{n}-1}$. Fix $m \in \mathbb{N}$ and let $y^{1}, y^{2}, \ldots, y^{m} \in B_{l_{n}-2 k-2 d}$; we now associate with this list a unique element of $B_{m\left(l_{n}-k\right)}$. Choose $s^{1}, s^{2}, \ldots, s^{m}, t^{1}, t^{2}, \ldots, t^{m} \in B_{d}$ so that the word $w:=$ $y^{1} s^{1} x t^{1} y^{2} s^{2} x t^{2} \cdots t^{m-1} y^{m} s^{m} x t^{m} \in\{1,2, \ldots, l\}^{m\left(l_{n}-k\right)}$ is such that $t^{m} u \in \Sigma$; this is possible as $A^{d}>0$.

It is easy to see that as $x$ is contained in any subword of length $n$, the word $z_{0} z_{1} \cdots z_{l_{n}-1}$ cannot be contained as a subword of the periodic extension of $w$. Hence, $w \in B_{m}\left(l_{n}-k\right), l_{n}$ and so

$$
Z_{m\left(l_{n}-k\right), l_{n}}(\phi) \geq\left(a e^{\left(l_{n}-2 k-2 d\right)(P(\phi)-\epsilon)}\right)^{m}>\left(a e^{l_{n}(1-\epsilon)(P(\phi)-\epsilon)}\right)^{m} .
$$


Taking logarithms, dividing by $m$, and letting $m \rightarrow \infty$ yields

$$
P_{\tilde{\Sigma}_{n}}(\phi) \geq \frac{\log (a)}{l_{n}-k}+(1-\epsilon) \frac{l_{n}}{l_{n}-k}(P(\phi)-\epsilon) .
$$

Finally, letting $n \rightarrow \infty$ and $\epsilon \rightarrow 0$ gives the result.

Remark 3.9. The proof of Proposition 3.8 is modified from [4], where an analogous result for topological entropy is proved.

3.3. A uniform Lasota-Yorke inequality. We now prove that the transfer operators $\mathcal{L}_{n}$ satisfy a uniform Lasota-Yorke inequality. We assume that the transfer operator $\mathcal{L}$ is normalized, i.e. $\mathcal{L} 1=1$. Iterating the perturbed operator $\mathcal{L}_{n}$, we see that

$$
\left(\mathcal{L}_{n}^{k} w\right)(x)=\sum_{\sigma^{k}(y)=x} h_{n, k}(y) e^{\phi^{k}(y)} w(y),
$$

where $h_{n, k}(x)=\prod_{j=0}^{k-1} \chi_{U_{n}^{c}}\left(\sigma^{j} x\right)$ and $\phi^{k}(y)=\sum_{j=0}^{k-1} \phi\left(\sigma^{j}(y)\right)$.

LEMMA 3.10. For any positive integers $k, n$, we have

$$
\left\|\mathcal{L}_{n}^{k}\right\|_{h} \leq 1
$$

Proof. Let $w \in L^{1}$; then

$$
\begin{aligned}
\left\|\mathcal{L}_{n} w\right\|_{1} & =\int\left|\mathcal{L}_{\chi_{U_{n}^{c}}} w\right| d \mu \\
& \leq \int \mathcal{L}\left|\chi_{U_{n}^{c}} w\right| d \mu \\
& =\int\left|\chi_{U_{n}^{c}} w\right| d \mu \leq\|w\|_{1} .
\end{aligned}
$$

In addition, fixing $j \geq 0, m \geq 1$, we see that

$$
\begin{aligned}
\theta^{-m} \int_{\sigma^{-j}\left(U_{m}\right)}\left|\mathcal{L}_{n} w\right| d \mu & \leq \theta^{-m} \int_{\sigma^{-j}\left(U_{m}\right)} \mathcal{L}\left(\left|\chi_{U_{n}^{c}} w\right|\right) d \mu \\
& =\theta^{-m} \int_{\sigma^{-(j+1)}\left(U_{m}\right)} \chi_{U_{n}^{c}}|w| d \mu \\
& \leq \theta^{-m} \int_{\sigma^{-(j+1)}\left(U_{m}\right)}|w| d \mu \leq|w|_{h} .
\end{aligned}
$$

Taking the supremum over $j$ and $m$ yields

$$
\left|\mathcal{L}_{n} w\right|_{h} \leq|w|_{h}
$$

Combining (4) and (5) and iterating completes the proof.

LEMMA 3.11. There exists a constant $c>0$ such that for any positive integers $n, k$ we have

$$
\left|h_{n, k} w\right|_{\theta} \leq|w|_{\theta}+c \theta^{-k}\|w\|_{h}
$$

for all $w \in \mathcal{B}_{\theta}$.

Proof. We prove this by induction, namely we prove that for any $w \in \mathcal{B}_{\theta}$ we have

$$
\left|\chi_{\sigma^{-j}\left(U_{n}^{c}\right)} w\right|_{\theta} \leq|w|_{\theta}+\theta^{-j}\|w\|_{h} .
$$


To show this, fix a positive integer $m$. We consider two cases, namely: $j+n \leq m$ and $m<j+n$. If we suppose that $j+n \leq m$, then $\operatorname{osc}\left(\chi_{\sigma^{-j}\left(U_{n}^{c}\right)} w, m, x\right) \leq \operatorname{osc}(w, m, x)$ for all $x \in \Sigma$, and thus

$$
\theta^{-m} \int \operatorname{osc}\left(\chi_{\sigma^{-j}\left(U_{n}^{c}\right)} w, m, x\right) d \mu(x) \leq \theta^{-m} \int \operatorname{osc}(w, m, x) d \mu(x) \leq|w|_{\theta} .
$$

On the other hand, if $m<j+n$ it is easy to see that if $[x]_{m} \subset \sigma^{-j}\left(U_{n}^{c}\right)$, then $\operatorname{osc}\left(\chi_{\sigma^{-j}\left(U_{n}^{c}\right)} w, m, x\right)=\operatorname{osc}(w, m, x)$. If, however, $[x]_{m} \cap \sigma^{-j}\left(U_{n}\right) \neq \emptyset$ then

$$
\operatorname{osc}\left(\chi_{\sigma^{-j}\left(U_{n}^{c}\right)} w, m, x\right)=\max \left(\operatorname{osc}(w, m, x),\left\|\chi_{[x]_{m}} w\right\|_{\infty}\right),
$$

in which case

$$
\begin{aligned}
\operatorname{osc}\left(\chi_{\sigma^{-j}\left(U_{n}^{c}\right)} w, m, x\right) & =\max \left(\operatorname{osc}(w, m, x),\left\|\chi_{[x]_{m}} w\right\|_{\infty}\right) \\
& \leq \operatorname{osc}(w, m, x)+\frac{1}{\mu[x]_{m}} \int_{[x]_{m}}|w| d \mu,
\end{aligned}
$$

which implies that

$$
\theta^{-m} \int \operatorname{osc}\left(\chi_{\sigma^{-j}\left(U_{n}^{c}\right)} w, m, x\right) d \mu(x) \leq|w|_{\theta}+\theta^{-m} \int_{\left\{x:[x]_{m} \cap \sigma^{-j}\left(U_{n}\right) \neq \emptyset\right\}}|w| d \mu .
$$

We now analyse two further subcases. If $m \leq j$, we see that

$$
\theta^{-m} \int_{\left\{x:[x]_{m} \cap \sigma^{-j}\left(U_{n}\right) \neq \emptyset\right\}}|w| d \mu \leq \theta^{-j}\|w\|_{1} .
$$

If $j<m<j+n$, the fact that the open sets $\left\{U_{n}\right\}_{n}$ are nested implies that

$$
\left\{x:[x]_{m} \cap \sigma^{-j}\left(U_{n}\right) \neq \emptyset\right\} \subset \sigma^{-j}\left(U_{m-j}\right) .
$$

In which case,

$$
\theta^{-m} \int_{\left\{x:[x]_{m} \cap \sigma^{-j}\left(U_{n}\right) \neq \emptyset\right\}}|w| d \mu \leq \theta^{-j}|w|_{h} .
$$

If we combine (7), (9), and (10), we obtain (6). This completes the proof.

LEMMA 3.12. There exists a constant $c>0$ such that

$$
\left\|\mathcal{L}_{n}^{k} w\right\|_{\theta} \leq c\left(\theta^{k}\|w\|_{\theta}+\|w\|_{h}\right)
$$

for all $w \in \mathcal{B}_{\theta}$ and $n, k \geq 1$.

Proof. Fix $x, y \in \Sigma$ and suppose that $d_{\theta}(x, y)=\theta^{m}$ with $m \geq 1$; then

$$
\begin{aligned}
\left|\left(\mathcal{L}_{n}^{k} w\right)(x)-\left(\mathcal{L}_{n}^{k} w\right)(y)\right| \leq & \sum_{|i|=k}\left|e^{\phi^{k}(i x)} h_{n, k}(i x) w(i x)-e^{\phi^{k}(i y)} h_{n, k}(i y) w(i y)\right| \\
\leq & \sum_{|i|=k} e^{\phi^{k}(i x)}\left|h_{n, k}(i x) w(i x)-h_{n, k}(i y) w(i y)\right| \\
& +e^{\phi^{k}(i x)}\left|1-e^{\phi^{k}(i y)-\phi^{k}(i x)}\right||w(i y)| \\
\leq & \sum_{|i|=k} e^{\phi^{k}(i x)}\left[\operatorname{osc}\left(h_{n, k} w, k+m, i x\right)+c \cdot \operatorname{osc}(w, k+m, i x)\right] \\
& +c \theta^{m} \sum_{|i|=k} \frac{e^{\phi^{k}(i x)}}{\mu[i x]_{k+m}} \int_{\mu[i x]_{k+m}}|w| d \mu .
\end{aligned}
$$


Integrating and dividing by $\theta^{m}$ implies that

$$
\left|\mathcal{L}_{n}^{k} w\right|_{\theta} \leq c \theta^{k}\left(|w|_{\theta}+\left|h_{n, k} w\right|_{\theta}\right)+c\|w\|_{1} .
$$

And so, from (4) and (11) along with Lemma 3.11, we deduce that

$$
\begin{aligned}
\left\|\mathcal{L}_{n}^{k} w\right\|_{\theta} & =\left|\mathcal{L}_{n}^{k} w\right|_{\theta}+\left\|\mathcal{L}_{n}^{k} w\right\|_{1} \\
& \leq c \theta^{k}\left(\left|h_{n, k} w\right|_{\theta}+|w|_{\theta}\right)+\|w\|_{1} \\
& \leq c \theta^{k}|w|_{\theta}+c\|w\|_{h} \leq c \theta^{k}\|w\|_{\theta}+c\|w\|_{h} .
\end{aligned}
$$

This completes the proof.

Remark 3.13. The advantage of introducing the weak norm $\|\cdot\|_{h}$ is that it overcomes the restrictions imposed by the usual weak norm $\|\cdot\|_{1}$. In particular, had we considered the usual $\|\cdot\|_{1}$ norm it would have imposed the condition that $0<\theta<1$ be chosen sufficiently small (leading to complications later in the proof when we also require that $\rho<\theta<1$ ).

3.4. Quasi-compactness of $L_{n}$. A prerequisite for proving quasi-compactness of $\mathcal{L}_{n}$ is that the unit ball is compact with respect to the weak norm.

Proposition 3.14. The set $B=\left\{w \in \mathcal{B}_{\theta}:\|w\|_{\theta} \leq 1\right\}$ is $\|\cdot\|_{h}$-compact.

Proof. Let $\left(f_{n}\right)_{n} \in B$ be any sequence. By Proposition 3.1, there exist a subsequence $\left(f_{n_{k}}\right)_{k}$ and $f \in B$ such that $\left\|f_{n_{k}}-f\right\|_{1} \rightarrow 0$. It suffices to show that $\left|f_{n_{k}}-f\right|_{h} \rightarrow 0$. As $f, f_{n_{k}} \in B$, we have that $c=\sup _{k \geq 1}\left\|f-f_{n_{k}}\right\|_{\infty}<\infty$. Fix $\epsilon>0$ and choose a positive integer $M$ such that $\theta^{-m} \mu\left(U_{m}\right) \leq \epsilon / c$ for all $m>M$. Choose a positive integer $K$ such that $\left\|f-f_{n_{k}}\right\|_{1} \leq \theta^{M} \epsilon$ for all $k \geq K$. For fixed $m, j$, if $m>M$ we have

$$
\theta^{-m} \int_{\sigma^{-j}\left(U_{m}\right)}\left|f-f_{n_{k}}\right| d \mu \leq \theta^{-m} \mu\left(U_{m}\right)\left\|f-f_{n_{k}}\right\|_{\infty}<\epsilon .
$$

Otherwise, $m \leq M$, in which case for $k \geq K$ we have

$$
\theta^{-m} \int_{\sigma^{-j}\left(U_{m}\right)}\left|f-f_{n_{k}}\right| d \mu \leq \theta^{-M} \int\left|f-f_{n_{k}}\right| d \mu<\epsilon .
$$

Taking (12) and (13) together implies that $\left|f-f_{n_{k}}\right|_{h}<\epsilon$ for $k \geq K$. This completes the proof.

We now prove quasi-compactness of $\mathcal{L}_{n}$ using a criterion of Hennion.

LEMMA 3.15. The essential spectral radii of the operators $\mathcal{L}_{n}$ are uniformly bounded by $\theta$.

Proof. To show that the essential spectral radii of the operators $\mathcal{L}_{n}$ are bounded by $\theta$, we note that Lemmas 3.12 and 3.14 show that the operators $\mathcal{L}_{n}$ satisfy the hypotheses of [10, Corollary 1], namely:

(1) $\mathcal{L}_{n}\left(\left\{w \in \mathcal{B}_{\theta}:\|w\|_{\theta} \leq 1\right\}\right)$ is conditionally compact in $\left(\mathcal{B}_{\theta},\|\cdot\|_{h}\right)$;

(2) for each $k$, there exist positive real numbers $R_{k}, r_{k}$ such that $\liminf _{k \rightarrow \infty}\left(r_{k}\right)^{1 / k}=$ $r<\lambda_{n}$ for which

$$
\left\|\mathcal{L}_{n}^{k}(w)\right\|_{\theta} \leq r_{k}\|w\|_{\theta}+R_{k}\|w\|_{h} \quad \text { for all } w \in \mathcal{B}_{\theta} .
$$


In which case, we conclude that $\mathcal{L}_{n}$ is quasi-compact with essential spectral radius bounded by $r$. Condition (1) can be deduced from Proposition 3.1, while condition (2) is the uniform Lasota-Yorke inequality proved in Lemma 3.12. Finally, Proposition 3.8 implies that for any $\theta \in(0,1)$ we have that $\lambda_{n}>\theta$ for large $n$.

3.5. Stability of the spectrum. We introduce a so-called 'asymmetric operator norm' for which the operators $\mathcal{L}_{n}$ converge to $\mathcal{L}$ as $n \rightarrow \infty$. For a linear operator $Q: \mathcal{B}_{\theta} \rightarrow \mathcal{B}_{\theta}$, we define

$$
\||Q|\|=\sup \left\{\|Q w\|_{h}:\|w\|_{\theta} \leq 1\right\} .
$$

Recall the Gibbs property (1) of $\mu$, namely that there exists a constant $c>1$ such that for any $x \in \Sigma$ and a positive integer $n$ we have that

$$
c^{-1} \leq \frac{\mu[x]_{n}}{e^{\phi^{n}(x)}} \leq c .
$$

Using the above, it is relatively easy to show the following proposition, which is stated without proof.

PROPOSITION 3.16. (Gibbs property) There exists a constant $c>0$ such that for any positive integers $n, m$ and $j \geq n$ we have

$$
\mu\left(U_{n} \cap \sigma^{-j}\left(U_{m}\right)\right) \leq c \mu\left(U_{n}\right) \mu\left(U_{m}\right) .
$$

LEMMA 3.17. There exists a constant $c>0$ such that

$$
\left\|\left|\mathcal{L}-\mathcal{L}_{n}\right|\right\| \leq c\left(\rho \theta^{-1}\right)^{n}
$$

for all $n$.

Proof. Let $w \in \mathcal{B}_{\theta}$ be such that $\|w\|_{\theta} \leq 1$; then

$$
\begin{aligned}
\left\|\left(\mathcal{L}-\mathcal{L}_{n}\right) w\right\|_{1} & =\left\|\mathcal{L} \chi_{U_{n}} w\right\|_{1} \\
& \leq\left\|\chi_{U_{n}} w\right\|_{1} \\
& \leq \mu\left(U_{n}\right)\|w\|_{\infty} \leq c \mu\left(U_{n}\right)\|w\|_{\theta} \leq c \mu\left(U_{n}\right) .
\end{aligned}
$$

On the other hand, for fixed $m, j$ we have

$$
\theta^{-m} \int_{\sigma^{-j}\left(U_{m}\right)}\left|\left(\mathcal{L}-\mathcal{L}_{n}\right) w\right| d \mu \leq c \theta^{-m} \mu\left(\sigma^{-(j+1)}\left(U_{m}\right) \cap U_{n}\right)\|w\|_{\theta} .
$$

Fix positive integers $m, j$. We study three cases, namely:

(1) $n \leq j+1$;

(2) $j+1<n<m+j+1$;

(3) $m+j+1 \leq n$.

First, we suppose that $n \leq j+1$, which implies from Proposition 3.16 that

$$
\theta^{-m} \mu\left(\sigma^{-(j+1)}\left(U_{m}\right) \cap U_{n}\right) \leq c \theta^{-m} \mu\left(U_{m}\right) \mu\left(U_{n}\right) \leq c \rho^{n} .
$$

Next, we suppose that $j+1<n<m+j+1$; then, observing that the nested property of $\left\{U_{n}\right\}_{n}$ gives us $\sigma^{-(j+1)}\left(U_{m}\right) \cap U_{n} \subset \sigma^{-(j+1)}\left(U_{m}\right) \cap U_{j+1}$, combining this 
with Proposition 3.16 we see that

$$
\begin{aligned}
\theta^{-m} \mu\left(\sigma^{-(j+1)}\left(U_{m}\right) \cap U_{n}\right) & \leq \mu\left(\sigma^{-(j+1)}\left(U_{m}\right) \cap U_{j+1}\right) \\
& \leq c \theta^{-m} \mu\left(U_{m}\right) \mu\left(U_{j+1}\right) \\
& \leq c \theta^{-m} \rho^{m+j+1} \\
& \leq c\left(\theta^{-1} \rho\right)^{m+j+1} \\
& \leq c\left(\theta^{-1} \rho\right)^{n} .
\end{aligned}
$$

If $n \geq m+j+1$,

$$
\theta^{-m} \mu\left(\sigma^{-(j+1)}\left(U_{m}\right) \cap U_{n}\right) \leq \theta^{-m} \mu\left(U_{n}\right) \leq \theta^{-n} \mu\left(U_{n}\right) \leq c\left(\theta^{-1} \rho\right)^{n} .
$$

Combining (16), (17), and (18) yields

$$
\left|\left(\mathcal{L}-\mathcal{L}_{n}\right) w\right|_{h} \leq c\left(\theta^{-1} \rho\right)^{n}\|w\|_{\theta} .
$$

Combining this with (15) completes the proof.

We note that Lemmas $3.10,3.12,3.15$, and 3.17 show that the operators $\mathcal{L}_{n}$ satisfy the hypotheses of [15, Theorem 1]. We now cite a specific consequence of the result.

For $\delta>0$ and $r>\theta$, let

$$
V_{\delta, r}=\{z \in \mathbb{C}:|z| \leq r \text { or } \operatorname{dist}(z, \operatorname{spec}(\mathcal{L})) \leq \delta\} .
$$

Then, by [15, Theorem 1], there exists $N=N(\delta, r)$ such that

$$
S_{\delta, r}=\sup \left\{\left\|\left(z-\mathcal{L}_{n}\right)^{-1}\right\|_{\theta}: n \geq N, z \in \mathbb{C} \backslash V_{\delta, r}\right\}<\infty,
$$

where $\left\|\left(z-\mathcal{L}_{n}\right)^{-1}\right\|_{\theta}$ denotes the operator norm of $\left(z-\mathcal{L}_{n}\right)^{-1}: \mathcal{B}_{\theta} \rightarrow \mathcal{B}_{\theta}$.

We may use quasi-compactness of $\mathcal{L}_{n}$ to write

$$
\mathcal{L}_{n}=\lambda_{n} E_{n}+\Psi_{n}
$$

where $E_{n}$ is a projection onto the eigenspace $\left\{c g_{n}: c \in \mathbb{C}\right\}$ and $E_{n} \Psi_{n}=\Psi_{n} E_{n}=0$.

PROPOSITION 3.18. There exist a positive integer $N$ and constants $c>0$ and $0<q<1$ such that for all $n \geq N$ we have

$$
\left\|\Psi_{n}^{k} 1\right\|_{\infty} \leq c q^{k} \quad \text { for any } k \geq 1
$$

Proof. Fix $q \in(\theta, 1)$ such that $\operatorname{spec}(\mathcal{L}) \backslash\{1\} \subset B(0, q)$. Then by Proposition 3.8 there exists a positive integer $N$ such that for all $n \geq N$, we may write using standard operator calculus

$$
\Psi_{n}^{k}=\frac{1}{2 \pi i} \int_{|t|=q} t^{k}\left(t-\mathcal{L}_{n}\right)^{-1} d t .
$$

Then, from Lemma 3.7 and (19) above, we see that

$$
\begin{aligned}
\left\|\Psi_{n}^{k} 1\right\|_{\infty} & \leq c\left\|\Psi_{n}^{k} 1\right\|_{\theta} \\
& \leq c \int_{|t|=q}|t|^{k}\left\|\left(t-\mathcal{L}_{n}\right)^{-1}\right\|_{\theta} d t \\
& \leq c q^{k} .
\end{aligned}
$$

Remark 3.19. This result (Proposition 3.18) is claimed in an article of Hirata [12]. However, the proof presented in the article contains an error which we correct in this section. In particular, this allows us to recover the exponential and Poisson return time estimates claimed in [12] for conformal expanding maps. 
PROPOSITION 3.20. There exists a constant $c>0$ such that for all $n$

Proof. For $n \geq N$, write

$$
\left\|E_{n} 1\right\|_{\infty} \leq c .
$$

$$
E_{n}=\frac{1}{2 \pi i} \int_{|t-1|=1-q}\left(t-\mathcal{L}_{n}\right)^{-1} d t .
$$

Then, from Lemma 3.7 and (19) above, we see that

$$
\begin{aligned}
\left\|E_{n} 1\right\|_{\infty} & \leq c\left\|E_{n} 1\right\|_{\theta} \\
& \leq c \int_{|t-1|=1-q}\left\|\left(t-\mathcal{L}_{n}\right)^{-1}\right\|_{\theta} d t \\
& \leq c .
\end{aligned}
$$

4. An asymptotic formula for $\lambda_{n}$

In this section, we prove the following proposition.

Proposition 4.1. Fix $\phi \in \mathcal{B}_{\theta}$; then

$$
\lim _{n \rightarrow \infty} \frac{\lambda-\lambda_{n}}{\mu\left(U_{n}\right)}= \begin{cases}\lambda & \text { if } z \text { is not periodic } \\ \lambda\left(1-\lambda^{-p} e^{\phi^{p}(z)}\right) & \text { if } z \text { has prime period } p .\end{cases}
$$

We prove the proposition in the case that $\mathcal{L}$ is normalized, i.e. $\mathcal{L} 1=1$; the more general statement above can be deduced by scaling the operator.

Let $m_{n}$ denote the restriction of $\mu$ to $I_{n}$, i.e.

$$
m_{n}=\frac{\left.\mu\right|_{U_{n}}}{\mu\left(U_{n}\right)} .
$$

The following four lemmas were motivated by corresponding results in [12].

LEMMA 4.2. If $z$ is non-periodic, then

$$
\lim _{n \rightarrow \infty} \frac{\int E_{n}\left(\mathcal{L} \chi_{U_{n}}\right) d m_{n}}{1-\lambda_{n}}=\lim _{n \rightarrow \infty} \int E_{n} 1 d m_{n}=1 .
$$

Proof. For simplicity, we put

$$
\left[E_{n}\right]=\int E_{n}\left(\mathcal{L} \chi_{U_{n}}\right) d m_{n} .
$$

Then, by using $\mathcal{L} \chi_{U_{n}}=1-\mathcal{L}_{n} 1$,

$$
\left[E_{n}\right]=\left(1-\lambda_{n}\right) \int E_{n} 1 d m_{n} .
$$

As $z$ is non-periodic, it follows from the fact that a countable intersection of nested compact sets is non-empty that for any integer $k \geq 1$, there exists $N_{k}$ such that $U_{N_{k}} \cap$ $\sigma^{-j}\left(U_{N_{k}}\right)=\emptyset$ for $j=1,2, \ldots, k$.

Then, for any $x \in \sigma^{-j} U_{N_{k}}, 1 \leq j \leq k$, we have that $x \notin U_{N_{k}}$. So, for any $n>N_{k}$ and any $x \in \sigma^{-k} U_{n}$, we see that

$$
\chi_{U_{n}^{c}}(x) \chi_{U_{n}^{c}}(\sigma(x)) \cdots \chi_{U_{n}^{c}}\left(\sigma^{k-1}(x)\right)=1 .
$$


So, for $n>N_{k}$, we see that

$$
\chi_{U_{n}}(x) \mathcal{L}_{n}^{k} 1(x)=\chi_{U_{n}}(x) \mathcal{L}^{k} 1(x)=\chi_{U_{n}}(x) .
$$

And so $\int \mathcal{L}_{n} 1 d m_{n}=1$ for all $n>N_{k}$.

We now use the decomposition $\mathcal{L}_{n}^{k}=\lambda_{n}^{k} E_{n}+\Psi_{n}^{k}$ to see that for any $k$ and $n>N_{k}$ we have

$$
\begin{aligned}
\left|1-\int E_{n} 1 d m_{n}\right| & =\left|\left(\lambda_{n}^{k}-1\right) \int E_{n} 1 d m_{n}+\int \Psi_{n}^{k} 1 d m_{n}\right| \\
& \leq\left|1-\lambda_{n}^{k}\right|\left\|E_{n} 1\right\|_{\infty}+\left\|\Psi_{n}^{k} 1\right\|_{\infty} \\
& \leq c\left(\left|1-\lambda_{n}^{k}\right|+q^{k}\right),
\end{aligned}
$$

where Propositions 3.18 and 3.20 were used in the final line. This completes the proof.

LEMMA 4.3. If $z$ is non-periodic, then

$$
\lim _{n \rightarrow \infty} \frac{\int E_{n}\left(\mathcal{L} \chi_{U_{n}}\right) d m_{n}}{\mu\left(U_{n}\right)}=1 .
$$

Proof. We let $T_{n}(x)$ denote the first return time (assuming that it exists) for $x \in U_{n}$, i.e.

$$
T_{n}(x)=\inf \left\{i \in \mathbb{N}: \sigma^{i}(x) \in U_{n}\right\} ;
$$

then

$$
\begin{aligned}
\int T_{n} d m_{n}= & \sum_{i=1}^{\infty} i m_{n}\left(T_{n}=i\right) \\
= & m_{n}\left(T_{n}=1\right)+\sum_{i=2}^{\infty} i \int \mathcal{L}_{n}^{i-1}\left(\mathcal{L}_{\chi_{U_{n}}}\right) d m_{n} \\
= & m_{n}\left(T_{n}=1\right)+\int E_{n}\left(\mathcal{L} \chi_{U_{n}}\right) d m_{n} \sum_{i=2}^{\infty} i \lambda_{n}^{i-1} \\
& +\sum_{i=2}^{\infty} i \int \Psi_{n}^{i-1}\left(\mathcal{L}_{n} \chi_{U_{n}}\right) d m_{n} \\
= & m_{n}\left(T_{n}=1\right)+\int E_{n}\left(\mathcal{L}_{\chi_{U_{n}}}\right) d m_{n}\left(\frac{1}{\left(1-\lambda_{n}\right)^{2}}-1\right) \\
& +\sum_{i=2}^{\infty} \int \Psi_{n}^{i-1} 1 d m_{n} .
\end{aligned}
$$

But, by Kac's theorem, $\int T_{n} d m_{n}=1 / \mu\left(U_{n}\right)$ and thus

$$
\begin{aligned}
\frac{\int E_{n}\left(\mathcal{L} \chi_{U_{n}}\right) d m_{n}}{\mu\left(U_{n}\right)}= & \underbrace{\left.\frac{\int E_{n}\left(\mathcal{L} \chi_{U_{n}}\right) d \mu_{n}}{1-\lambda_{n}}\right)^{2}}_{\rightarrow 1}+\underbrace{\int E_{n}\left(\mathcal{L} \chi_{U_{n}}\right) d m_{n}}_{\rightarrow 0} \\
& \times \underbrace{\left(m_{n}\left(T_{n}=1\right)-\int E_{n}\left(\mathcal{L} \chi_{U_{n}}\right) d m_{n}+\sum_{k=1}^{\infty} \Psi_{n}^{k} 1 d m_{n}\right)}_{=O(1)} .
\end{aligned}
$$

This completes the proof. 
LEMMA 4.4. If $z$ has prime period $p$, then

$$
\lim _{n \rightarrow \infty} \frac{\int E_{n}\left(\mathcal{L} \chi_{U_{n}}\right) d m_{n}}{1-\lambda_{n}}=\lim _{n \rightarrow \infty} \int E_{n} 1 d m_{n}=1-e^{\phi^{p}(z)} .
$$

Proof. Fix a large positive integer $t$ and set $k=p t$. We have for large $n$ that

$$
\begin{aligned}
\chi_{U_{n}}(x)-\chi_{U_{n}}(x) \mathcal{L}_{n}^{k} 1(x) & =\chi_{U_{n}}(x) \sum_{\sigma^{k}(y)=x} \chi_{\bigcup_{j=0}^{k-1} \sigma^{-j}\left(U_{n}\right)}(y) e^{\phi^{k}(y)} \\
& =\chi_{U_{n}}(x) \sum_{\sigma^{k}(y)=x} \chi_{\sigma^{k-p}\left[z_{0} z_{1} \cdots z_{p-1}\right]}(y) e^{\phi^{k}(y)} \\
& =\chi_{U_{n}}(x) \sum_{\sigma{ }^{p m}(y)=x} e^{\phi^{p m}(y)} \chi_{\sigma-p(m-1)}\left[z_{0} z_{1} \cdots z_{p-1}\right] \\
& =\chi_{U_{n}}(x) \mathcal{L}^{p m}\left(\chi_{\left[z_{0} z_{1} \cdots z_{p-1}\right]} \circ \sigma^{p(m-1)}\right)(x) \\
& =\chi_{U_{n}}(x) \mathcal{L}^{p}\left(\chi_{\left[z_{0} z_{1} \cdots z_{p-1}\right]}\right)(x),
\end{aligned}
$$

where assumption (5) on the family $\left\{U_{n}\right\}_{n}$ was utilized in the second line. Hence,

$$
\begin{aligned}
\left|1-e^{\phi^{p}(z)}-\int \mathcal{L}_{n}^{k} 1 d m_{n}\right| & \leq\left|\int \mathcal{L}^{p}\left(\chi_{\left[z_{0} z_{1} \cdots z_{p-1}\right]}\right)(x)-e^{\phi^{p}(z)} d m_{n}(x)\right| \\
& \leq \sup _{y \in[z]_{n+p}}\left|\phi^{p}(y)-\phi^{p}(z)\right| \\
& \leq \frac{|\phi|_{\theta, \infty}}{1-\theta} \operatorname{diam}\left(U_{n}\right) \rightarrow 0 \quad(n \rightarrow \infty),
\end{aligned}
$$

where $|\cdot|_{\theta, \infty}$ denotes the usual Hölder semi-norm.

Hence, for any $k=p t$,

$$
\lim _{n \rightarrow \infty} \int \mathcal{L}_{n}^{k} 1 d m_{n}=1-e^{\phi^{p}(z)}
$$

On the other hand, by Lemma 4.2, for large $n$

$$
\left|\int \mathcal{L}_{n}^{k} 1 d m_{n}-\lambda_{n}^{k} \int E_{n} d m_{n}\right|=\left|\int \Psi_{n}^{k} 1 d m_{n}\right| \leq\left\|\Psi_{n}^{k}\right\|_{\infty} \leq c q^{k} .
$$

We fix $k=p t$ and $\lambda_{n} \rightarrow 1$ as $n \rightarrow \infty$; hence,

$$
\lim _{n \rightarrow \infty} \int E_{n} 1 d m_{n}=1-e^{\phi^{p}(z)}
$$

LEMMA 4.5. If $z$ has prime period $p$, then

$$
\lim _{n \rightarrow \infty} \frac{\int E_{n}\left(\mathcal{L} \chi_{U_{n}}\right) d m_{n}}{\mu\left(U_{n}\right)}=\left(1-e^{\phi^{p}(z)}\right)^{2} .
$$

Proof. The proof of this is a combination of the methods from Lemma 4.3 and the result of Lemma 4.4.

Combining Lemmas 4.2, 4.3, 4.4, and 4.5 proves Proposition 4.1. 


\section{Escape rates for Gibbs measures}

In this section, we prove the analogue of Theorem 1.1 in the setting of a topologically mixing subshift of finite type, namely, we prove the following theorem.

THEOREM 5.1. Suppose that the $\left\{U_{n}\right\}_{n}$ satisfy assumptions (1)-(5). Let $\phi: \Sigma \rightarrow \mathbb{R}$ be Hölder continuous and let $\mu$ denote the associated equilibrium state; then

$$
\lim _{n \rightarrow \infty} \frac{r_{\mu}\left(U_{n}\right)}{\mu\left(U_{n}\right)}= \begin{cases}1 & \text { if } z \text { is not periodic }, \\ 1-e^{\phi^{p}(z)-p P(\phi)} & \text { if } z \text { has prime period } p,\end{cases}
$$

where $\phi^{p}(z)=\phi(z)+\phi(\sigma(z))+\cdots+\phi\left(\sigma^{p-1}(z)\right)$.

It is well known that the escape rate $r_{\mu}\left(U_{n}\right)$ is related to the spectral radius $\lambda_{n}$ and we include the proof of the following proposition only for completeness.

PROPOSITION 5.2. We have the following relation:

$$
r_{\mu}\left(U_{n}\right)=-\log \left(\lambda_{n}\right)
$$

Proof. We can write

$$
\begin{aligned}
\mu\left\{x \in \Sigma: \sigma^{i}(x) \notin U_{n}, 0 \leq i \leq k-1\right\} & =\int\left(\prod_{i=0}^{k-1} \chi_{U_{n}^{c}}\left(\sigma^{i} x\right)\right) d \mu(x) \\
& =\int \mathcal{L}^{k}\left(\prod_{i=0}^{k-1} \chi_{U_{n}^{c}}\left(\sigma^{i} x\right)\right) d \mu(x) \\
& =\int \mathcal{L}_{n}^{k} 1(x) d \mu(x) \\
& =\lambda_{n}^{k} \int E_{n} 1 d \mu+\int \Psi_{n}^{k} 1 d \mu .
\end{aligned}
$$

Using Propositions 3.18 and 3.20, we see that

$$
r_{\mu}\left(U_{n}\right)=\lim _{k \rightarrow \infty}-\frac{1}{k} \log \mu\left\{x \in \Sigma: \sigma^{i}(x) \notin U_{n}, 0 \leq i \leq k-1\right\}=-\log \left(\lambda_{n}\right) .
$$

We now prove Theorem 5.1.

Proof. We assume without loss of generality that $P(\phi)=0$. In which case, we see from Proposition 5.2 that

$$
\begin{aligned}
\frac{r_{\mu}\left(U_{n}\right)}{\mu\left(U_{n}\right)} & =\frac{-\log \left(\lambda_{n}\right)}{\mu\left(U_{n}\right)} \\
& =\frac{\log (\lambda)-\log \left(\lambda_{n}\right)}{\mu\left(U_{n}\right)} \\
& =\frac{\lambda-\lambda_{n}}{\mu\left(U_{n}\right)} \frac{\log (\lambda)-\log \left(\lambda_{n}\right)}{\lambda-\lambda_{n}} .
\end{aligned}
$$

The result now follows from Proposition 4.1. 
We also can obtain results relating to the convergence of the topological pressure.

THEOREM 5.3. Suppose that the $\left\{U_{n}\right\}_{n}$ satisfy assumptions (1)-(5). Let $\phi: \Sigma \rightarrow \mathbb{R}$ be Hölder continuous and let $\mu$ denote the associated equilibrium state; then

$$
\lim _{n \rightarrow \infty} \frac{P(\phi)-P_{\Sigma_{n}}(\phi)}{\mu\left(U_{n}\right)}= \begin{cases}1 & \text { if } z \text { is not periodic }, \\ 1-e^{\phi^{p}(z)-p P(\phi)} & \text { if } z \text { has prime period } p .\end{cases}
$$

Proof. Using $\lambda=e^{P(\phi)}$, we see that

$$
\frac{P(\phi)-P_{\Sigma_{n}}(\phi)}{\mu\left(U_{n}\right)}=\frac{P(\phi)-P_{\Sigma_{n}}(\phi)}{e^{P(\phi)}-e^{P_{\Sigma_{n}}(\phi)}} \frac{\lambda-\lambda_{n}}{\mu\left(U_{n}\right)} .
$$

Observing that

$$
\lim _{n \rightarrow \infty} \frac{P(\phi)-P_{\Sigma_{n}}(\phi)}{e^{P(\phi)}-e^{P_{\Sigma_{n}}(\phi)}}=e^{-P(\phi)},
$$

and combining this, (21), and Proposition 4.1 completes the proof.

An immediate corollary is the following.

COROllary 5.4. Let $\mu$ denote the measure of maximal entropy (i.e. the Parry measure [20]); then

$$
\lim _{n \rightarrow \infty} \frac{\left.h_{\mathrm{top}}(\sigma)-h_{\mathrm{top}}\left(\left.\sigma\right|_{\Sigma_{n}}\right)\right)}{\mu\left(U_{n}\right)}= \begin{cases}1 & \text { if } z \text { is not periodic, } \\ 1-e^{-p h_{\mathrm{top}}(\sigma)} & \text { if } z \text { has prime period } p .\end{cases}
$$

Remark 5.5. The rate of convergence of the topological entropy of the restriction of the shift to these sets was studied by Lind [18] who proved, in the case that the $U_{n}$ consisted of a single cylinder of length $n$, i.e. $U_{n}=[z]_{n}$, the existence of a constant $c>1$ such that

$$
1 / c \leq \frac{h_{\text {top }}(\sigma)-h_{\text {top }}\left(\left.\sigma\right|_{\Sigma_{n}}\right)}{\mu\left(U_{n}\right)} \leq c \text { for all } n .
$$

\section{Proof of Theorem 1.1}

In this section, we prove Theorem 1.1. Let $\mathcal{M}$ be a Riemannian manifold and $f: \mathcal{M} \rightarrow \mathcal{M}$ a $C^{1}$-map. Let $J$ be a compact subset of $\mathcal{M}$ such that $f(J)=J$. We say that the pair $(J, f)$ is a conformal repeller if:

(1) $\left.f\right|_{J}$ is a conformal map;

(2) there exist $c>0$ and $\lambda>1$ such that $\left\|d f_{x}^{n} v\right\| \geq c \lambda^{n}\|v\|$ for all $x \in J, v \in T_{x} \mathcal{M}$, and $n \geq 1$;

(3) $f$ is topologically mixing on $J$;

(4) $J$ is maximal, i.e. there exists an open neighbourhood $V \supset J$ such that

$$
J=\left\{x \in V: f^{n}(x) \in V \text { for all } n \geq 0\right\} .
$$

Let $\phi: J \rightarrow \mathbb{R}$ be $\alpha$-Hölder and let $\mu$ denote the associated equilibrium state. For an open set $U \subset J$, we let $r_{\mu}(U)$ denote the escape rate of $\mu$ through $U$.

It is well known that an expanding map has a finite Markov partition $\left\{R_{1}, R_{2}, \ldots, R_{l}\right\}$, and that there exists a continuous semi-conjugacy $\pi: \Sigma \rightarrow J$, where $\Sigma$ is a subshift of finite type on $l$ symbols. By choosing $\lambda^{-\alpha}<\theta<1$ and considering $\Sigma$ equipped with the metric $d_{\theta}$, it can be seen that the map $\tilde{\phi}=\phi \circ \pi: \Sigma \rightarrow \mathbb{R}$ is $d_{\theta}$-Lipshitz, and so $\tilde{\phi} \in \mathcal{B}_{\theta}$. 
We state without proof the following result of Bowen [2].

PROPOSITION 6.1. (Bowen) There exists a positive integer $d$ such that the cardinality of $\pi^{-1}(x)$ is at most $d$ for all $x \in J$.

This proposition was used to prove the following corollary.

COROLlaRY 6.2. (Bowen) $x \in \Sigma$ is periodic if and only if $\pi(x) \in J$ is periodic.

We also require the following technical lemmas.

Lemma 6.3. For any periodic point $z \in J$, there exists a Markov partition $\left\{R_{1}, R_{2}, \ldots\right.$, $\left.R_{m}\right\}$ such that $z \in \bigcup_{i=1}^{m} \operatorname{int}\left(R_{i}\right)$.

Proof. This follows easily from the standard construction of Markov partitions (using shadowing); for example see [27].

LEMMA 6.4. There exist constants $s, c_{1}>0$ such that $\mu(B(z, \epsilon)) \leq c_{1} \epsilon^{s}$ for all $\epsilon>0$.

Proof. Let $\tilde{\phi}: \Sigma \rightarrow \mathbb{R}$ be defined by $\tilde{\phi}(x)=\phi(\pi(x))$, and denote the associated equilibrium state by $\tilde{\mu}$; then $\mu=\pi^{*}(\tilde{\mu})$.

For $\epsilon>0$, let $\mathcal{U}_{\epsilon}$ denote the Moran cover associated with the Markov partition $\left\{R_{1}\right.$, $\left.R_{2}, \ldots, R_{m}\right\}$ (see [23, p. 200]). Then for $z \in J$ we choose elements $U_{1}, U_{2}, \ldots, U_{k} \in \mathcal{U}_{\epsilon}$ which intersect $B(z, \epsilon)$. A basic property of Moran covers is that:

(1) $U_{i}=\pi\left[z_{0}^{i} z_{1}^{i} \cdots z_{n\left(z^{i}\right)}^{i}\right]$, where $z^{i} \in \Sigma$;

(2) $\operatorname{diam}\left(U_{i}\right) \leq \epsilon<\operatorname{diam}\left(\pi\left[z_{0}^{i} z_{1}^{i} \cdots z_{n\left(z^{i}\right)-1}^{i}\right]\right)$;

(3) $k \leq K$, where $K$ is independent of both $z$ and $\epsilon$.

In which case, it suffices to show that $\mu\left(U_{i}\right) \leq c \epsilon^{s}$ for some constant $c>0$. To see this, we observe that a basic property of Gibbs measures is that for any $x \in \Sigma$, there exist $c>0$ and $\gamma \in(0,1)$ such that $\tilde{\mu}[x]_{n} \leq c \gamma^{n}$ for $n=1,2, \ldots$ In addition to $f \in C^{1+\alpha}$ and conformal, we have that $c \lambda^{-n\left(z^{i}\right)} \leq \epsilon$ for any $\epsilon>0$. In which case, we see that

$$
\mu(B(z, \epsilon)) \leq \sum_{i=1}^{k} \mu\left(U_{i}\right)=\sum_{i=1}^{k} \tilde{\mu}\left[z_{0}^{i} z_{1}^{i} \cdots z_{\left.n_{(} z^{i}\right)}^{i}\right] \leq K c^{1+\log (\gamma) / \log (\lambda)} \epsilon^{-\log (\gamma) / \log (\lambda)} .
$$

Next, we require the so-called ' $D$-annular decay property', that is, there exists a constant $c_{2}>0$ such that for all $x \in J, \epsilon>0$ and $0<\delta<1$, we have that

$$
\mu(B(x, \epsilon) \backslash B(x,(1-\delta) \epsilon)) \leq c_{2} \delta^{D} \mu(B(x, \epsilon)) .
$$

A related condition is the 'doubling' or 'Federer' property, namely there exists a constant $K>1$ such that for all $x \in J$ and $\epsilon>0$, we have

$$
\mu(B(x, 2 \epsilon)) \leq K \mu(B(x, \epsilon)) .
$$

Evidently, a measure that satisfies the $D$-annular decay property also satisfies the doubling property. The converse was shown by Buckley in [5, Corollary 2.2]. In the context of an equilibrium state $\mu$ supported on a conformal repeller, Pesin and Weiss [22] showed that $\mu$ satisfies the doubling property. We collect these two results in the following proposition. 
Proposition 6.5. There exists a $D$ such that $\mu$ satisfies the D-annular decay property.

We now prove Theorem 1.1.

Proof. We first prove the result if $z \in J$ is not periodic. As a consequence of Proposition 6.1 we have that $\pi^{-1}\{z\}=\left\{z^{1}, z^{2}, \ldots, z^{r}\right\}$. Further, Corollary 6.2 implies that each $z^{i}$ is non-periodic.

Hence, to show Theorem 1.1 it suffices to show that

$$
\lim _{\epsilon \rightarrow 0} \frac{r_{\tilde{\mu}}\left(\pi^{-1}(B(z, \epsilon))\right)}{\tilde{\mu}\left(\pi^{-1}(B(z, \epsilon))\right)}=1 .
$$

First, we observe that Theorem 5.1 may be modified to accommodate multiple non-periodic points appearing in the intersection; this modification is trivial and we therefore omit the proof. For non-periodic points, the new hypotheses become:

(1) let $\left\{V_{n}\right\}$ be a family of nested sets with each $V_{n}$ being a finite union of cylinders. Suppose further that $\bigcap_{n \geq 1} V_{n}$ consists of finitely many non-periodic points $\left\{z^{1}, z^{2}, \ldots, z^{r}\right\}$;

(2) there exist constants $c>0$ and $0<\rho<1$ such that $\tilde{\mu}\left(V_{n}\right) \leq c \rho^{k_{n}}$ for all $n \geq 1$; here $k_{n}$ denotes the maximum length of a cylinder in $V_{n}$;

(3) There exist a sequence $\left(l_{n}\right)_{n}$ and a constant $\kappa>0$ such that $\kappa<l_{n} / k_{n} \geq 1$ and $V_{n} \subset \bigcup_{i=1}^{r}\left[z^{i}\right]_{l_{n}}$ for all $n \geq 1$.

If the sets $\left\{V_{n}\right\}_{n}$ satisfy these hypotheses, then we conclude that

$$
\lim _{n \rightarrow \infty} \frac{r_{\tilde{\mu}}\left(V_{n}\right)}{\tilde{\mu}\left(V_{n}\right)}=1 .
$$

For $\epsilon>0$ and a positive integer $k$, we set

$$
U_{k, \epsilon}=\left\{U \in \bigvee_{i=0}^{k-1} f^{-i} \mathcal{R}: U \cap B(z, \epsilon) \neq \emptyset\right\} .
$$

We observe that due to $f$ being uniformly expanding, there exist constants $c_{3}>0$ and $0<\rho<1$ such that

$$
\operatorname{diam}(U) \leq c_{3} \rho^{k}
$$

for any $U \in U_{k, \epsilon}$.

Let $\delta_{k}=c_{3} \rho^{k} /\left(\epsilon+c_{3} \rho^{k}\right)$, in which case it is easy to see that

$$
\bigcup_{U \in U_{k, \epsilon}} U \subset B\left(z, \epsilon+c_{3} \rho^{k}\right)=B\left(z,\left(1-\delta_{k}\right)^{-1} \epsilon\right) .
$$

Fix $\eta>0$ small and choose $k=k(\epsilon, \eta)$ such that

$$
\rho^{k} \leq \frac{\epsilon}{c_{3}\left(\left(c_{2} \eta^{-1}\right)^{1 / D}-1\right)}<\rho^{k-1},
$$

in which case we see that

$$
\begin{aligned}
(1-\eta) \mu\left(\bigcup_{U \in U_{k, \epsilon}} U\right) & \leq\left(1-c_{2} \delta_{k}^{D}\right) \mu\left(\bigcup_{U \in U_{k, \epsilon}} U\right) \\
& \leq\left(1-c_{2} \delta_{k}^{D}\right) \mu\left(B\left(z,\left(1-\delta_{k}\right)^{-1} \epsilon\right)\right) \\
& \leq \mu(B(z, \epsilon)),
\end{aligned}
$$


where the $D$-annular decay property was used in the final line. Now let $\left\{\epsilon_{n}\right\}_{n}$ be any monotonic sequence with $\epsilon_{n} \rightarrow 0$ and set

$$
U_{n}=\bigcup_{U \in U_{k\left(\epsilon_{n}, \eta\right), \epsilon_{n}}} U
$$

Observing that $U_{n}$ is a finite union of $k_{n}:=k\left(\epsilon_{n}, \eta\right)$ th level refinements of the Markov partition, there exists $V_{n} \subset \Sigma$, a finite union of cylinders of length $k_{n}$, such that $\pi\left(V_{n}\right)=U_{n}$

We claim that $V_{n}$ satisfies the hypotheses of the modified Theorem 5.1. Clearly, the $V_{n}$ are nested (1), so it suffices to show that $\tilde{\mu}\left(V_{n}\right)$ decays exponentially in $n$. To see this, we observe that

$$
\begin{aligned}
\tilde{\mu}\left(V_{n}\right) & =\mu\left(U_{n}\right) \\
& \leq(1-\eta)^{-1} \mu\left(B\left(z, \epsilon_{n}\right)\right) \\
& \leq c_{1} \epsilon_{n}^{s} \leq c_{1}\left(c_{3}\left(\left(c_{2} \eta^{-1}\right)^{1 / D}-1\right)\right)^{s} \rho^{s\left(k_{n}-1\right)} .
\end{aligned}
$$

And, thus, we see that $\tilde{\mu}\left(V_{n}\right)$ decreases exponentially in $k_{n}$, which proves (2).

As $f$ is conformal and $z \in\left[z^{i}\right]_{l}$ for all $i$ and $l$, there exist constants $c_{4}>0$ and $0<\varrho<1$ such that for any $i \in\{1,2, \ldots, r\}$ and $l \in \mathbb{N}$, we have that $c_{4}^{-1} \leq \operatorname{diam}\left(\pi\left[z^{i}\right]_{l}\right) / \varrho^{l}$. Let $l_{n}$ be the minimum such $l$ such that $c_{4}^{-1} \varrho^{l} \geq 2 \epsilon_{n}$. It is easy to see that for such a choice of $l$, we have that $V_{n} \subset \bigcup_{i=1}^{r}\left[z^{i}\right]_{l_{n}}$. In addition, we have that $l_{n}>c_{5} k_{n}$ for some constant $c_{5}>0$; this proves (3). Thus, we deduce from the modified Theorem 5.1

$$
\lim _{n \rightarrow \infty} \frac{r_{\tilde{\mu}}\left(V_{n}\right)}{\tilde{\mu}\left(V_{n}\right)}=1 .
$$

And, so, by monotonicity of escape rates and (23), we see that

$$
\begin{aligned}
\limsup _{n \rightarrow \infty} \frac{r_{\mu}\left(B\left(z, \epsilon_{n}\right)\right)}{\mu\left(B\left(z, \epsilon_{n}\right)\right)} & \leq(1-\eta)^{-1} \limsup _{n \rightarrow \infty} \frac{r_{\mu}\left(U_{n}\right)}{\mu\left(U_{n}\right)} \\
& =(1-\eta)^{-1} \limsup _{n \rightarrow \infty} \frac{r_{\tilde{\mu}}\left(V_{n}\right)}{\tilde{\mu}\left(V_{n}\right)}=(1-\eta)^{-1} .
\end{aligned}
$$

Similarly, using the same method we may obtain a lower bound, which in conjunction with (24) gives

$$
\lim _{n \rightarrow \infty} \frac{r_{\mu}\left(B\left(z, \epsilon_{n}\right)\right)}{\mu\left(B\left(z, \epsilon_{n}\right)\right)}=1 .
$$

We now turn our attention to the case where $z$ is periodic. By Lemma 6.3, we may assume that $\pi^{-1}(z)$ consists of a single point of prime period $p$, say $\pi\left(z^{\prime}\right)=z$.

As before, we approximate $B(z, \epsilon)$ from outside using elements of $\bigvee_{i=0}^{k-1} f^{-i} \mathcal{R}$, which may be thought of as cylinders of length $k$ in a subshift of finite type. Recall the hypotheses for Theorem 5.1:

(1) let $\left\{V_{n}\right\}$ be a family of nested sets with each $V_{n}$ being a finite union of cylinders. Suppose further that $\bigcap_{n \geq 1} V_{n}=\left\{z^{\prime}\right\}$, where $z^{\prime}$ has prime period $p$;

(2) there exist constants $c>0$ and $0<\rho<1$ such that $\tilde{\mu}\left(V_{n}\right) \leq c \rho^{k_{n}}$ for $n=1,2, \ldots$; here $k_{n}$ denotes the maximum length of a cylinder in $V_{n}$;

(3) for each $n \geq 1$, we have that $\sigma^{-p}\left(V_{n}\right) \cap\left[z_{0}^{\prime} z_{1}^{\prime} \cdots z_{p-1}^{\prime}\right] \subset V_{n}$. 
In which case, we deduce from Theorem 5.1 that

$$
\lim _{n \rightarrow \infty} \frac{r_{\tilde{\mu}}\left(V_{n}\right)}{\tilde{\mu}\left(V_{n}\right)}=1-e^{\tilde{\phi}^{p}\left(z^{\prime}\right)} .
$$

We first approximate $B\left(z, \epsilon_{n}\right)$ from outside using the same method employed previously. For $\eta>0$, we obtain $U_{n} \supset B\left(z, \epsilon_{n}\right)$ nested, each being a finite union of elements from $\bigvee_{i=0}^{k(n)-1} f^{-i} \mathcal{R}$ for some $k$, with the property that $\mu\left(U_{n}\right) \leq(1-\eta)^{-1}$ $\mu\left(B\left(z, \epsilon_{n}\right)\right)$. As before, we may find a $V_{n} \subset \Sigma$ which is a finite union of cylinders. It is easy to see that $V_{n}$ satisfies conditions (1) and (2). To see (3), we observe that for $\epsilon_{n}$ small, expansivity of $f$ and the fact that $z$ has prime period $p$ yields $f^{-p}\left(B\left(z, \epsilon_{n}\right)\right) \cap$ $\pi\left[z_{0}^{\prime}, z_{1}^{\prime}, \ldots, z_{p-1}^{\prime}\right] \subset B\left(z, \epsilon_{n}\right)$. A simple argument extends this to approximations of balls centred on $z$. Using monotonicity of escape rates together with the conclusions of Theorem 5.1 yields

$$
\begin{aligned}
\limsup _{n \rightarrow \infty} \frac{r_{\mu}\left(B\left(z, \epsilon_{n}\right)\right)}{\mu\left(B\left(z, \epsilon_{n}\right)\right)} & \leq(1-\eta)^{-1} \limsup _{n \rightarrow \infty} \frac{r_{\mu}\left(U_{n}\right)}{\mu\left(U_{n}\right)} \\
& =(1-\eta)^{-1} \limsup _{n \rightarrow \infty} \frac{r_{\tilde{\mu}}\left(V_{n}\right)}{\tilde{\mu}\left(V_{n}\right)} \\
& =(1-\eta)^{-1}\left(1-e^{\tilde{\phi}^{p}}\left(z^{\prime}\right)\right) \\
& =(1-\eta)^{-1}\left(1-e^{\phi^{p}(z)}\right) .
\end{aligned}
$$

Similarly, using the same method we may obtain a lower bound, which, in conjunction with (25), yields

$$
\lim _{n \rightarrow \infty} \frac{r_{\mu}\left(B\left(z, \epsilon_{n}\right)\right)}{\mu\left(B\left(z, \epsilon_{n}\right)\right)}=1-e^{\phi^{p}(z)}
$$

\section{Proof of Theorem 1.2}

In this section, we study the asymptotic behaviour of the Hausdorff dimension of the nontrapped set. Let $f: J \rightarrow J$ be a conformal repeller as defined in the previous section; we make the further assumption that $f \in C^{1+\alpha}(J)$ for some $\alpha>0$. Fix $z \in J$. For $\epsilon>0$, we define

$$
J_{\epsilon}=\left\{x \in J: f^{k}(x) \notin B(z, \epsilon), \text { for all } k \geq 0\right\},
$$

i.e. all points whose orbits are $\epsilon$-bounded away from $z$.

Let $\mu$ denote the equilibrium state related to the potential $\psi=-s \log \left|f^{\prime}\right|$, where $s=\operatorname{dim}_{H}(J)$. As before, we may study the escape rate $r_{\mu}(B(z, \epsilon))$ of $\mu$ through $B(z, \epsilon)$ and its associated asymptotic, i.e.

$$
d_{\phi}(z):=\lim _{\epsilon \rightarrow 0} \frac{r_{\mu}(B(z, \epsilon))}{\mu(B(z, \epsilon))} .
$$

The method of proof is as follows: in a similar vein to the proof of Theorem 1.1, we first prove the result where the hole consists of a finite union of refinements of the Markov partition and then extend it to the case of geometric balls via an approximation argument.

Let $\mathcal{R}=\left\{R_{1}, R_{2}, \ldots, R_{m}\right\}$ denote a Markov partition for the conformal repeller $J$; this induces a semi-conjugacy $\pi$ between a subshift of finite type $(\Sigma, \sigma)$ and the conformal 
repeller $(J, f)$. Let $I_{n} \in \bigvee_{i=0}^{n-1} f^{-j} \mathcal{R}$ be a nested family such that $\bigcap_{n \geq 0} I_{n}=\{z\}$. We let $J_{n}$ denote the set of points in $J$ which do not fall down the hole $I_{n}$, i.e.

$$
J_{n}=\left\{x \in J: f^{k}(x) \notin I_{n}, \text { for all } k \geq 0\right\} .
$$

Let $s_{\epsilon}$ denote the Hausdorff dimension of the set $J_{\epsilon}$.

PROPOSITION 7.1. Under the assumptions above,

$$
\lim _{n \rightarrow \infty} \frac{s-s_{n}}{\mu\left(I_{n}\right)}=\frac{d_{\phi}(z)}{\int \log \left|f^{\prime}\right| d \mu} .
$$

A crucial ingredient to the proof of Proposition 7.1 is the following result of Ruelle [24].

PROPOSITION 7.2. (Ruelle) Let $s \geq 0$ be the unique real number for which $P\left(-s \log \left|f^{\prime}\right|\right)=0$; then $\operatorname{dim}_{H}(J)=s$.

Let $\tilde{\phi}(x):=-\log \left|f^{\prime}(\pi(x))\right|$. It is easy to see that the semi-conjugacy $\pi$ being oneone on a set of full measure for all equilibrium states for Hölder potentials implies that the Hausdorff dimension of $J$ is the unique real number $s$ for which $P(s \tilde{\phi})=0$. A similar argument shows that $\operatorname{dim}_{H}\left(J_{n}\right)=s_{n}$, where $s_{n}$ is the unique real number satisfying $P_{\Sigma_{n}}\left(s_{n} \tilde{\phi}\right)=0$. We may therefore translate the problem into the language of subshifts of finite type. As the family $\left\{I_{n}\right\}$ is nested, there exists a point $z^{\prime} \in \Sigma$ such that $\pi\left[z^{\prime}\right]=I_{n}$. Accordingly, if we set

$$
\Sigma_{n}=\left\{x \in \Sigma: \sigma^{k}(x) \notin\left[z^{\prime}\right]_{n} \text { for } k=0,1,2, \ldots\right\},
$$

then $\pi\left(\Sigma_{n}\right)=J_{n}$. Let $\tilde{\phi}(x)=-\log \left|f^{\prime}(\pi(x))\right|$; then it is easy to see that the semiconjugacy $\pi$ being one-one on a set of full measure for all equilibrium states for Hölder potentials implies that the Hausdorff dimension of $J$ is the unique real number $s$ for which $P(s \tilde{\phi})=0$. A similar argument shows that $\operatorname{dim}_{H}\left(J_{n}\right)=s_{n}$, where $s_{n}$ is the unique real number satisfying $P_{\Sigma_{n}}\left(s_{n} \tilde{\phi}\right)=0$. We therefore may prove the result in the setting of subshifts of finite type.

For $t \geq 0$, we let $\mathcal{L}_{t}: \mathcal{B}_{\theta} \rightarrow \mathcal{B}_{\theta}$ denote the transfer operator associated with the potential $t \tilde{\phi}$, i.e.

$$
\left(\mathcal{L}_{t} w\right)(x)=\sum_{\sigma(y)=x} \frac{w(y)}{\left|f^{\prime}(\pi(y))\right|^{t}} ;
$$

analogously, we define the perturbed transfer operator $\mathcal{L}_{t, n}: \mathcal{B}_{\theta} \rightarrow \mathcal{B}_{\theta}$ to be $\left(\mathcal{L}_{t, n} w\right)(x)=$ $\left(\mathcal{L}_{t} \chi_{\left[z^{\prime}\right]_{n}^{c}} w\right)(x)$. We let $g_{t}$ (respectively $\left.g_{t, n}\right)$ and $v_{t}$ (respectively $v_{t, n}$ ) denote the eigenfunctions and eigenmeasures guaranteed by Proposition 3.2 applied to $\mathcal{L}_{t}$ (respectively $\mathcal{L}_{t, n}$ ). We shall assume without loss of generality that $\int g_{t} d v_{t}=\int g_{t, n} d v_{t, n}=1$ for all $t \geq 0$ and $n \geq 1$. The associated equilibrium states will be denoted by $\mu_{t}$ and $\mu_{t, n}$, observing that one can show that $d \mu_{t}=g_{t} d v_{t}$ (respectively $d \mu_{t, n}=g_{t, n} d v_{t, n}$ ). We proved earlier that both $\mathcal{L}_{t}$ and $\mathcal{L}_{t, n}$ have spectral gaps; we denote their maximal eigenvalues by $\lambda_{t}$ and $\lambda_{t, n}$, respectively. As $\log \left(\lambda_{t}\right)=P(t \tilde{\phi})$ (respectively $\log \left(\lambda_{t, n}\right)=$ $P_{\Sigma_{n}}(t \tilde{\phi})$ ), the problem of finding the Hausdorff dimensions of $J$ (respectively $J_{n}$ ) reduces to finding the values of $t$ (respectively $t_{n}$ ) such that $\lambda_{t}=1$ (respectively $\lambda_{t_{n}, n}=1$ ).

The proof of Proposition 7.1 relies on a few elementary facts: the maps $t \mapsto \lambda_{t, n}$ are analytic and non-increasing in $t$, while for a fixed $t$ the sequence $\left\{\lambda_{t, n}\right\}_{n}$ is increasing 
(and converges to $\lambda_{t}$ ), we use Taylor's theorem applied to $\lambda_{t, n}$ about $t=s$ to obtain an approximation of $\lambda_{t, n}$ close to $\lambda_{s, n}$, we then use Theorem 5.1 and let $n \rightarrow \infty$ to prove the result. The main problem then reduces to analysing the behaviour of the first $\lambda_{t, n}^{\prime}=d / d t\left(\lambda_{t, n}\right)$ and second $\lambda_{t, n}^{\prime \prime}=d^{2} / d t^{2}\left(\lambda_{t, n}\right)$ derivatives of $\lambda_{t, n}$, which is the focus of the following two technical lemmas.

LEMMA 7.3. For any $t \geq 0$, we have that $\lim _{n \rightarrow \infty} \lambda_{t, n}^{\prime}=\lambda_{t}^{\prime}$.

Proof. We first obtain an explicit formula for $\lambda_{t, n}^{\prime}$; to do this, we follow an argument of Ruelle [25, p. 96, Exercise 5] to prove that for any $t \geq 0$ and $n=1,2, \ldots$,

$$
\lambda_{t, n}^{\prime}=-\lambda_{t, n} \int \log \left|f^{\prime}\right| d \mu_{t, n}
$$

Analogously, for the unperturbed operator,

$$
\lambda_{t}^{\prime}=-\lambda_{t} \int \log \left|f^{\prime}\right| d \mu_{t}
$$

To see this, we take the eigenfunction equation

$$
L_{t, n} g_{t, n}=\lambda_{t, n} g_{t, n}
$$

Differentiating once yields

$$
L_{t, n}^{\prime} g_{t, n}+L_{t, n} g_{t, n}^{\prime}=\lambda_{t, n}^{\prime} g_{t, n}+\lambda_{t, n} g_{t, n}^{\prime},
$$

and then integrating with respect to $v_{t, n}$ and cancelling terms yields

$$
\lambda_{t, n}^{\prime}=\int \mathcal{L}_{t, n}^{\prime}\left(g_{t, n}\right) d v_{t, n}=\int \mathcal{L}_{t, n}\left(\phi g_{t, n}\right) d v_{t, n}=\lambda_{t, n} \int \phi d \mu_{t, n}
$$

where $\phi=-\log \left|f^{\prime}\right|$. This shows (26); the proof of (27) is analogous and is omitted.

Without loss of generality, we may assume that $g_{t}=1$, that is, $\mathcal{L}_{t} 1(x)=\lambda_{t}$. We decompose the transfer operators $\mathcal{L}_{t, n}$ and $\mathcal{L}_{t}$ as

$$
\mathcal{L}_{t, n}=\lambda_{t, n} E_{t, n}+\Psi_{t, n}, \quad \mathcal{L}_{t}=\lambda_{t} E_{t}+\Psi_{t},
$$

where $E_{t, n}$ and $E_{t}$ are projection operators given by

$$
E_{t, n} w=\int w d v_{t, n} g_{t, n}, \quad E_{t} w=\int w d v_{t}
$$

and $\Psi_{t, n}$ (respectively $\Psi_{t}$ ) has a spectral radius strictly less than $\lambda_{t, n}$ (respectively $\lambda_{t}$ ).

From [15], we have that $\lim _{n \rightarrow \infty}\left\|\left|E_{t, n}-E_{t}\right|\right\|=0$ and so

$$
\begin{aligned}
\left\|g_{t, n}-g_{t}\right\|_{1} & \leq\left\|g_{t, n}-g_{t}\right\|_{h} \\
& =\left\|\left(E_{t, n}-E_{t}\right)(1)\right\|_{h} \leq\left\|\left|E_{t, n}-E_{t}\right|\right\|\|1\|_{\theta} \rightarrow 0 .
\end{aligned}
$$

Finally, to show that $\lambda_{t, n}^{\prime} \rightarrow \lambda_{t}^{\prime}$, it suffices to show that $E_{t, n}\left(g_{t, n} \phi\right) \rightarrow E_{t}(\phi)$. We first show that there exists a constant $c>0$ such that $\left\|g_{t, n} \phi\right\|_{\theta} \leq c$ for all $n$. We note 
that by [15, Corollary 1] there exist a constant $c>0$ and a positive integer $N$ such that $\left\|E_{t, n} w\right\|_{\theta} \leq c\left\|E_{t} w\right\|_{h}$ for any $w \in \mathcal{B}_{\theta}$ and $n \geq N$. In which case,

$$
\begin{aligned}
\left\|g_{t, n} \phi\right\|_{\theta} & =\left\|g_{t, n} \phi\right\|_{\theta}+\left\|g_{t, n} \phi\right\|_{1} \\
& \leq\left\|g_{t, n}\right\|_{\infty}|\phi|_{\theta}+\left|g_{t, n}\right|_{\theta}\|\phi\|_{\infty}+\|\phi\|_{\infty}\left\|g_{t, n}\right\|_{1} \\
& \leq 2\|\phi\|_{\theta}\left\|g_{t, n}\right\|_{\theta}+\|\phi\|_{\infty}\left\|g_{t, n}\right\|_{h} \\
& =2\|\phi\|_{\theta}\left\|E_{t, n} 1\right\|_{\theta}+\|\phi\|_{\infty}\left\|g_{t, n}\right\|_{h} \\
& \leq 2 c\|\phi\|_{\theta}\left\|E_{t, n} 1\right\|_{h}+\|\phi\|_{\infty}\left\|g_{t, n}\right\|_{h} \\
& =\left(2 c\|\phi\|_{\theta}+\|\phi\|_{\infty}\right)\left\|g_{t, n}\right\|_{h} .
\end{aligned}
$$

We observe that $\left\|g_{t, n}-1\right\|_{1} \rightarrow 0$ implies that $\left\|g_{t, n}-1\right\|_{h} \rightarrow 0$ and so $\left\|g_{t, n}\right\|_{\theta}$ is bounded. Next, we note that

$$
\begin{aligned}
\left\|E_{t, n}\left(g_{t, n} \phi\right)-E_{t}(\phi)\right\|_{1} & =\left\|\left|E_{t, n}-E_{t}\right|\right\|\left\|g_{t, n} \phi\right\|_{\theta}+\left\|E_{t}\left(\phi\left(g_{t, n}-1\right)\right)\right\|_{1} \\
& \leq c\left\|\left|E_{t, n}-E_{t}\right|\right\|+\|\phi\|_{\infty}\left\|E_{t}\right\|_{1}\left\|g_{t, n}-1\right\|_{1} .
\end{aligned}
$$

Both terms tend to zero by (30) and [15]. This completes the proof.

LEMmA 7.4. For any $s>0$, there exists $\delta>0$ such that $\sup _{n \geq 1} \sup _{t \in(s-\delta, s+\delta)} \lambda_{t, n}^{\prime \prime}<\infty$.

Proof. We first obtain an expression for $\lambda_{t, n}^{\prime \prime}$. Fix a positive integer $N$. Taking the eigenfunction equation $\mathcal{L}_{t, n}^{N} g_{t, n}=\lambda_{t, n}^{N} g_{t, n}$ and differentiating twice, integrating with respect to $v_{t, n}$, and cancelling yields

$$
\begin{aligned}
\lambda_{t, n}^{-1} \lambda_{t, n}^{\prime \prime}= & \frac{1}{N}\left[\int\left(\phi^{N}\right)^{2} g_{t, n} d v_{t, n}-N(N-1)\left(\lambda_{t, n}^{-1} \lambda_{t, n}^{\prime}\right)^{2}\right] \\
& +2\left[\frac{1}{N} \int \phi^{N} g_{t, n}^{\prime} d v_{t, n}-\lambda_{t, n}^{-1} \lambda_{t, n}^{\prime} \int g_{t, n}^{\prime} d v_{t, n}\right] .
\end{aligned}
$$

We observe that as $d \mu_{t, n}=g_{t, n} d v_{t, n}$ is strong mixing, this second term tends to zero as $N \rightarrow \infty$, and thus

$$
\lambda_{t, n}^{-1} \lambda_{t, n}^{\prime \prime}=\lim _{N \rightarrow \infty} \frac{1}{N}\left[\int\left(\phi^{N}\right)^{2} g_{t, n} d v_{t, n}-N(N-1)\left(\lambda_{t, n}^{-1} \lambda_{t, n}^{\prime}\right)^{2}\right] .
$$

We now estimate the term $N^{-1} \int\left(\phi^{N}\right)^{2} g_{t, n} d v_{t, n}$ : expanding the term $\left(\phi^{N}\right)^{2}$ and using the dual identity $\mathcal{L}_{t, n}^{*}\left(v_{t, n}\right)=\lambda_{t, n} v_{t, n}$ yields for $n$ large enough

$$
\begin{aligned}
N^{-1} \int\left(\phi^{N}\right)^{2} g_{t, n} d v_{t, n} & =\sum_{i=0}^{N-1} \sum_{j=0}^{N-1} \int g_{t, n} \phi \circ \sigma^{i} \phi \circ \sigma^{j} d v_{t, n} \\
& =\|\phi\|_{2}^{2}+\frac{2}{N} \sum_{k=0}^{N-1}(N-k) \int g_{t, n} \phi \phi \circ \sigma^{k} d v_{t, n} \\
& =\|\phi\|_{2}^{2}+\frac{2}{N} \sum_{k=0}^{N-1}(N-k) \lambda_{t, n}^{-k} \int \mathcal{L}_{t, n}^{k}\left(g_{t, n} \phi\right) \phi d v_{t, n}
\end{aligned}
$$


We apply the decomposition $L_{t, n}=\lambda_{t, n} E_{t, n}+\Psi_{t, n}$ along with Proposition 3.18 to (32) to obtain

$$
\begin{aligned}
N^{-1} \int\left(\phi^{N}\right)^{2} g_{t, n} d v_{t, n}= & \|\phi\|_{2}^{2}+\frac{2}{N} \sum_{k=0}^{N-1}(N-k) \\
& \times \int E_{t, n}\left(g_{t, n} \phi\right) \phi+\lambda_{t, n}^{-k} \Psi_{t, n}^{k}\left(g_{t, n} \phi\right) \phi d v_{t, n} \\
= & \|\phi\|_{2}^{2}+(N+1)\left(\int \phi g_{t, n} d v_{t, n}\right)^{2} \\
& +\frac{2}{N} \sum_{k=1}^{N-1}(N-k) \lambda_{t, n}^{-k} \int \Psi_{t, n}^{k}\left(g_{t, n} \phi\right) \phi d v_{t, n} .
\end{aligned}
$$

We note that $\left(\int \phi g_{t, n} d v_{t, n}\right)^{2}=\left(\lambda_{t, n}^{-1} \lambda_{t, n}^{\prime}\right)^{2}$ and so combining (31) and (33) we obtain

$$
\lambda_{t, n}^{-1} \lambda_{t, n}^{\prime \prime}=\|\phi\|_{2}^{2}+2\left(\lambda_{t, n}^{-1} \lambda_{t, n}^{\prime}\right)^{2}+\lim _{N \rightarrow \infty} \frac{2}{N} \sum_{k=0}^{N-1}(N-k) \lambda_{t, n}^{-k} \int \Psi_{t, n}^{k}\left(g_{t, n} \phi\right) \phi d v_{t, n} .
$$

Finally, we observe that the perturbation $t \mapsto \mathcal{L}_{t}$ is analytic and so for any $q>0$ such that $\operatorname{spec}\left(\mathcal{L}_{S}\right) \backslash\left\{\lambda_{s}\right\} \subset B(0, q)$, there exist a positive integer $M$ and $\delta>0$ such that $\lambda_{t, n}>q$ and $\operatorname{spec}\left(\mathcal{L}_{t, n}\right) \backslash\left\{\lambda_{t, n}\right\} \subset B(0, q)$ for all $n \geq M$ and $t \in(s-\delta, s+\delta)$. Combining this observation with Proposition 3.18 completes the proof.

We now prove Proposition 7.1.

Proof. We begin by proving that $s-s_{n}=O\left(\mu\left(I_{n}\right)\right)$; to see this, we observe that the map $t \mapsto \lambda_{t, n}$ is analytic, and so using Taylor's theorem we may write

$$
\lambda_{s_{n}, n}=1=\lambda_{s, n}+\lambda_{\xi_{n}, n}^{\prime}\left(s_{n}-s\right)
$$

for some $\xi_{n} \in\left(s_{n}, s\right)$. We note that Proposition 4.1 and Lemma 7.3 prove the claim. Next, we use Taylor's theorem once again to see that

$$
\lambda_{s_{n}, n}=1=\lambda_{s, n}+\lambda_{s, n}^{\prime}\left(s_{n}-s\right)+\lambda_{\xi_{n}, n}^{\prime \prime} O\left(\mu\left(I_{n}\right)^{2}\right)
$$

for $\xi_{n} \in\left(s_{n}, s\right)$. Rearranging yields

$$
\frac{s-s_{n}}{\mu\left(I_{n}\right)}=\frac{1}{-\lambda_{s, n}^{\prime}}\left[\frac{1-\lambda_{s, n}}{\mu\left(I_{n}\right)}+\lambda_{\xi_{n}, n}^{\prime \prime} O\left(\mu\left(I_{n}\right)\right)\right] .
$$

Finally, we let $n \rightarrow \infty$, observing that the right-hand side converges by Lemmas 7.3 and 7.4. This completes the proof.

We note that as in the case of escape rates Proposition 7.1 generalizes easily to the case of finite unions of symbolic holes. We now prove Theorem 1.2.

Proof. Let $\left\{\epsilon_{n}\right\}_{n}$ be any monotonic sequence with $\epsilon_{n} \rightarrow 0$. Fix $\eta>0$ and choose $U_{n} \subset$ $B\left(z, \epsilon_{n}\right) \subset V_{n}$ which consist of finite unions of refinements of the Markov partition $\mathcal{R}$ such that

$$
(1-\eta) \mu\left(V_{n}\right) \leq \mu\left(B\left(z, \epsilon_{n}\right)\right) \leq(1+\eta) \mu\left(U_{n}\right) .
$$


From the proof of Theorem 1.1, it is clear that we may choose the families $\left\{U_{n}\right\}_{n}$ and $\left\{V_{n}\right\}_{n}$ so that they satisfy the hypotheses of Proposition 7.1. Let $\underline{s}_{n}$ (respectively $\bar{s}_{n}$ ) denote the Hausdorff dimension of the non-trapped set with respect to the hole $U_{n}$ (respectively $V_{n}$ ). Monotonicity of the Hausdorff dimension along with (35) yields

$$
\frac{1}{1+\eta} \frac{s-\underline{s}_{n}}{\mu\left(U_{n}\right)} \leq \frac{s-s_{n}}{\mu\left(B\left(z, \epsilon_{n}\right)\right)} \leq \frac{1}{1-\eta} \frac{s-\bar{s}_{n}}{\mu\left(V_{n}\right)}
$$

for any $n$; combining this with Proposition 7.1 and letting $\eta \rightarrow 0$ completes the proof.

Acknowledgement. We would like to thank the referee for his careful reading of the original version of this paper and his numerous comments.

\section{REFERENCES}

[1] W. Bahsoun and C. Bose. Quasi-invariant measures, escape rates and the effect of the hole. Discrete Contin. Dyn. Syst. 27(3) (2010), 1107-1121.

[2] R. Bowen. Markov partitions and minimal sets for Axiom A diffeomorphisms. Amer. J. Math. 92 (1970), 907-918.

[3] R. Bowen. Equilibrium States and the Ergodic Theory of Anosov Diffeomorphisms, revised edn (Lecture Notes in Mathematics, 470). Springer, Berlin, 2008.

[4] M. Boyle, D. Lind and D. Rudolph. The automorphism group of a shift of finite type. Trans. Amer. Math. Soc. 306(1) (1988), 71-114.

[5] S. M. Buckley. Is the maximal function of a Lipschitz function continuous? Ann. Acad. Sci. Fenn. Math. 24(2) (1999), 519-528.

[6] L. A. Bunimovich and C. P. Dettmann. Peeping at chaos: nondestructive monitoring of chaotic systems by measuring long-time escape rates. Europhys. Lett. EPL 80(4) (2007), 6, Art. 40001.

[7] L. A. Bunimovich and A. Yurchenko. Where to place a hole to achieve fastest escape rate. Israel J. Math. to appear.

[8] P. Collet, S. Martínez and B. Schmitt. The Pianigiani-Yorke measure for topological Markov chains. Israel J. Math. 97 (1997), 61-70.

[9] M. F. Demers and L.-S. Young. Escape rates and conditionally invariant measures. Nonlinearity 19(2) (2006), 377-397.

[10] H. Hennion. Sur un théorème spectral et son application aux noyaux lipchitziens. Proc. Amer. Math. Soc. 118(2) (1993), 627-634.

[11] D. Hensley. Continued fraction Cantor sets, Hausdorff dimension, and functional analysis. J. Number Theory 40(3) (1992), 336-358.

[12] M. Hirata. Poisson law for Axiom A diffeomorphisms. Ergod. Th. \& Dynam. Sys. 13(3) (1993), 533-556.

[13] C. T. Ionescu Tulcea and G. Marinescu. Théorie ergodique pour des classes d'opérations non complètement continues. Ann. of Math. (2) 52 (1950), 140-147.

[14] G. Keller. Generalized bounded variation and applications to piecewise monotonic transformations. Z. Wahrsch. Verw. Gebiete. 69(3) (1985), 461-478.

[15] G. Keller and C. Liverani. Stability of the spectrum for transfer operators. Ann. Sc. Norm. Super. Pisa Cl. Sci. (4) 28(1) (1999), 141-152.

[16] G. Keller and C. Liverani. Rare events, escape rates and quasistationarity: some exact formulae. J. Stat. Phys. 135(3) (2009), 519-534.

[17] A. Lasota and J. A. Yorke. On the existence of invariant measures for piecewise monotonic transformations. Trans. Amer. Math. Soc. 186 (1973), 481-488.

[18] D. A. Lind. Perturbations of shifts of finite type. SIAM J. Discrete Math. 2(3) (1989), 350-365.

[19] C. Liverani and V. Maume-Deschamps. Lasota-Yorke maps with holes: conditionally invariant probability measures and invariant probability measures on the survivor set. Ann. Inst. H. Poincaré Probab. Statist. 39(3) (2003), 385-412. 
[20] W. Parry. Intrinsic Markov chains. Trans. Amer. Math. Soc. 112 (1964), 55-66.

[21] W. Parry and M. Pollicott. Zeta functions and the periodic orbit structure of hyperbolic dynamics. Astérisque (187-188) (1990), 1-268.

[22] Y. Pesin and H. Weiss. A multifractal analysis of equilibrium measures for conformal expanding maps and Moran-like geometric constructions. J. Stat. Phys. 86(1-2) (1997), 233-275.

[23] Y. B. Pesin. Dimension Theory in Dynamical Systems (Chicago Lectures in Mathematics). University of Chicago Press, Chicago, IL, 1997.

[24] D. Ruelle. Repellers for real analytic maps. Ergod. Th. \& Dynam. Sys. 2(1) (1982), 99-107.

[25] D. Ruelle. Thermodynamic formalism. Cambridge Mathematical Library, 2nd edn. Cambridge University Press, Cambridge, 2004.

[26] P. Walters. An Introduction to Ergodic Theory (Graduate Texts in Mathematics, 79). Springer, New York, 1982.

[27] M. Zinsmeister. Thermodynamic Formalism and Holomorphic Dynamical Systems (SMF/AMS Texts and Monographs, 2). American Mathematical Society, Providence, RI, 2000. 